\title{
An assessment of third-party reporting of close ties to measure sensitive behaviors: the Confidante Method to measure abortion incidence in Ethiopia and Uganda
}

\author{
Margaret Giorgio $\mathrm{PhD}^{1}$, Elizabeth Sully $\mathrm{PhD}^{1}$, Doris W. Chiu BA ${ }^{1}$ \\ ${ }^{1}$ Guttmacher Institute \\ 125 Maiden Lane, $7^{\text {Th }}$ Floor \\ New York, New York 10038 \\ January 12,2021
}

\begin{abstract}
Indirect estimation techniques are an important tool for measuring sensitive and stigmatized behaviors. This includes third-party reporting methods, which have become increasingly common in the field of abortion measurement, where direct survey approaches notoriously lead to underreporting. This paper provides the first in-depth assessment of one of the most widely used of these techniques in the field of abortion measurement: the Confidante Method. We outline six key assumptions behind the Confidante Method and describe how violations of these assumptions can bias resulting estimates. Using data from modules added to the nationally representative Performance Monitoring for Action (PMA) surveys in Uganda and Ethiopia in 2018, we compute one-year abortion incidence estimates using the Confidante Method. We also perform a validation check, using the method to estimate IUD and implant use. While our results reveal that the method performed differently in each country, there were implementation problems in both settings. Several of the method's foundational assumptions were violated, and efforts to adjust for these violations either failed or only partially addressed the resulting bias. Our validation check also failed, resulting in a gross over-estimate of IUD and implant use. We identify substantial biases in both the numerator and denominator of our abortion estimates and challenge the continued use of this method to estimate abortion incidence. These results have implications more broadly for the potential biases that can be introduced in using third-party reporting of close ties to measure other sensitive or stigmatized behaviors.
\end{abstract}




\section{Introduction}

Accurate estimates of the incidence of induced abortion are essential in order to track trends in fertility and contraception use. They also provide a necessary foundation for understanding the conditions under which abortions occur, risk factors for severe abortion complications, and unintended pregnancy rates. However, abortion is notoriously difficult to measure. In countries with restrictive abortion laws, information on abortion is not routinely gathered (Singh et al., 2018). In countries where abortion is legal yet highly stigmatized, the clandestine circumstances under which many abortions are performed make official records incomplete. Further, respondents are often reluctant to directly admit to having had an abortion in a survey (Jones and Forrest 1992; Jones and Kost 2007; Lindberg et al. 2020), and efforts to encourage direct reporting through anonymized response methods have failed to consistently generate reliable abortion incidence estimates (Juarez et al., 2010; Moseson et al., 2017; Oliveras \& Letamo, 2010).

As a result of these challenges, researchers have tended to rely on indirect estimation techniques for producing more robust abortion incidence estimates. One common indirect approach is the Abortion Incidence Complications Method (AICM) (Singh et al., 2010).The AICM uses data on patients hospitalized with induced abortion complications in combination with estimates of the proportion of all abortions that do not lead to facility-based treatment to calculate an abortion incidence rate. However, recent increases in the use of medication abortion likely limit the AICM's ability to accurately measure abortion incidence, as self-managed abortions become safer and reduce interactions with the formal healthcare system.

Another promising set of indirect methods for measuring abortion incidence are social network-based methods that use third party reporting (TRP) to collect information on abortions within a respondent's social network. TPR methods represent an improvement over the AICM, as they are not reliant on interactions with the health care system. These methods include (1) Anonymous Third Party Reporting (ATPR), which collects information on all close friends (Rossier, 2010; Rossier et al., 2006; Sedgh et al., 2011); (2) the Best Friend Approach, which collects information on a respondent's one closest female friend (or relative) (Yeatman \& Trinitapoli, 2011), and (3) the Confidante Method, which collects information on two to three women with whom the respondent reciprocally shares personal information 
(Sedgh \& Keogh, 2019). ${ }^{1}$ Based on the proliferation of the use of these methods to measure abortion, with the recent increase in use the of the Confidante Method in particular, there is a pressing need to evaluate the assumptions underlying these methods, potential violations of those assumptions, and the resulting biases in abortion estimates.

This paper provides the first critical assessment of the Confidante Method to measure abortion. We outline six key assumptions behind the Confidante Method and describe how potential violations of these assumptions can introduce bias to both the numerator and the denominator of these estimates. Using data from modules added to the nationally representative Performance Monitoring for Action (PMA) surveys in Uganda and Ethiopia in 2018, we employ the Confidante Method to compute one-year abortion incidence estimates, which we then compare to incidence estimates derived from self-reported abortions in the same surveys, as well as the most recent AICM abortion incidence estimate in each country. Ethiopia and Uganda represent different social and legal contexts for abortion; while abortion is largely stigmatized in both countries, safe abortion care is easily accessible in Ethiopia yet highly restricted in Uganda. This contrast provides further insight into how and why violations to assumptions may occur in different settings. We propose and test techniques to adjust for potential sources of bias in the Confidante Method. We also perform a validation check, using the method to estimate intrauterine device (IUD) and implant use, which can be reliably estimated through direct survey methods. Our findings highlight the serious challenges of using the Confidante Method to estimate abortion incidence, which has important implications for the use of this method to measure other sensitive and stigmatized behaviors.

\section{Background}

There is a long history of the use of TPR of close ties to measure hidden behaviors or events. Early examples of this are the Sibling Method (and its sisterhood subset), which were developed to estimate mortality; respondents are asked for an exhaustive list of siblings (or sisters) born to the same mother, for whom they provide information about gender, age, and survival status (Bicego, 1997; Gakidou \& King, 2006; Graham et al., 1989, 1989; Hill \& Trussell, 1977; Rutenberg \& Sullivan, 1991; Timæus \& Jasseh, 2004). Originally proposed by Hill and Trussell (1977), it is now one of the key methods for measuring maternal mortality in countries without accurate vital registration systems and has been included as a

\footnotetext{
${ }^{1}$ We recognize that non-binary individuals, transgender men, and people of other genders are capable of becoming pregnant and have abortions. However, the surveys we discuss in this paper that have implemented ATPR, the Best Friend Method, and the Confidante Method use recruitment strategies that rely on respondents self-identifying as cisgender female or cisgender women, as well as social tie definitions that use the terms "female" and "women" to define which social network members can be included in the surrogate samples. As such, throughout this paper we refer to respondent and surrogate samples as being composed of women.
} 
module in the Demographic and Health Surveys in 46 countries. Given its widespread use and longevity, there also exists a robust body of research interrogating its potential biases. This has included selection effects through survivor bias, biases introduced from sibship with no surviving siblings, recall errors in deaths and age at death, and reporting errors in sibship listings (Gakidou \& King, 2006; Helleringer et al., 2014; Masquelier et al., 2021; Stanton et al., 2000; Trussell \& Rodriguez, 1990). Corrections have been proposed to adjust for these biases, including a weighting adjustment and a corrected sibling survival (Gakidou \& King, 2006; Obermeyer et al., 2010), which has since been criticized for potentially overestimating mortality (Masquelier, 2013).

More recently, several indirect social network-based approaches that build off the Sibling Method have been developed to measure abortion incidence. The general assumptions shared by these methods is that respondents will be more willing to report others' abortions than to report their own, and respondents' social networks can be used to generate a surrogate sample. The first application of one such indirect network-based approach was the ATPR method (Rossier et al., 2006). Similar to the Sibling Method, the ATPR asks for an exhaustive listing of close friends and then asks whether each friend had an abortion (Rossier, 2010; Rossier et al., 2006; Sedgh et al., 2011). The abortion rate is calculated as the rate among all reported close friends. A key benefit of this method is that information is collected on multiple close ties, creating a large denominator. However, the denominator is sensitive to whether the respondent gives an accurate listing of women who fit the close tie definition, which could potentially bias the ATPR estimate. Previous applications of the ATPR have provided mixed results of its effectiveness; while a 2001 application of ATPR in Burkina Faso produced a rate that was comparable to other estimates in the region at the time, a 2009 study in Burkina Faso as well as a similar study in India produced unreasonably low abortion rates (Rossier, 2003, 2010; Sedgh et al., 2011).

The Best Friend Method similarly asks about abortions within respondents' social networks. However, each respondent is only asked to report on her one closest female friend (or relative) (Yeatman \& Trinitapoli, 2011). While this method corrects for the potential inaccurate listing limitation of the ATPR method, it results in a much smaller sample size. Also, the assumption that a sample comprised of each respondent's one "best friend" is representative of reproductive-age women may not be defensible if reported "best friends" have different abortion experiences than that of the general population. The Best Friend Method was employed in Malawi, where it yielded higher estimates of abortion than self-reports, suggesting better but possibly incomplete reporting (Yeatman and Trinitapoli 2011). 
The Confidante Method was designed to combine the strengthens of the ATPR and the Best Friend methods while making small adjustments to address their limitations (Sedgh \& Keogh, 2019). It asks each respondent to think of all of the women they know who would share their secrets with her, and with whom she would also share such secrets (a reciprocally-defined relationship). Then, instead of asking respondents to report information on all of these women (as in the ATPR), respondents are only asked to report on a fixed number of women with whom they are the closest. ${ }^{2}$ Fixing the number of Confidantes to a set maximum increases the sample size relative to the Best Friend Method while also precluding respondents from having to produce an accurate list of all women who fit the tie definition. In addition, the ATPR and Best Friend Method allow for the possibility that multiple respondents will report on the same person. If abortion is either negatively or positively correlated with "popularity", the resulting incidence estimates would be biased. For example, a study on reported close ties in a closed social network study on Likoma Island, Malawi, found popularity bias among men who had multiple sexual partnerships, potentially limiting the utility of this method for estimating that sensitive behavior (Helleringer et al., 2019). The Confidante Method's stipulation of reciprocity in the tie definition is designed to help diminish this potential for "popular" confidantes to be repeatedly included in the sample.

There has been a proliferation of TPR methods to measure abortions in recent years. The ATPR and Best Friend Method have been used to estimate abortion incidence in Burkina Faso (Rossier et al., 2006; Sedgh et al., 2011) and Malawi (Yeatman \& Trinitapoli, 2011). The Confidante Method has been tested even more broadly, including in Côte D’Ivoire (Bell, Sheehy, et al., 2020), Ghana (Keogh et al., 2020), India (Bell, et al. 2019), Indonesia (Stillman et al., 2020), Kinshasa, DRC (Ishoso et al., 2019), and Nigeria (Bell, et al. 2020). Given the fact that all three of these methods share several potential biases and limitations that have been well documented in the Sibling Method literature, an in-depth examination of the methods' assumptions and associated biases is needed in order to determine the validity of the resulting incidence estimates. Some recent work has begun to describe the assumptions of the Confidante Method, yet several key assumptions were missing (Bell, Shankar, et al., 2020). Another previous study raised concerns about sampling bias in third party reporting using a unique complete network dataset, but this work did not look at non-sampling biases or errors that can emerge that are specific to abortion reporting (Helleringer et al., 2019). Our analysis is the first to comprehensively examine the underlying assumptions associated with the use of TPR of close ties to measure abortion incidence, the biases to which violations in these assumptions could lead, and possible adjustments that can be used to correct for such biases.

\footnotetext{
${ }^{2}$ Recent applications of the Confidante Method have limited this number to two or three close ties (Bell, Shankar, et al., 2020; Keogh et al., 2020; Stillman et al., 2020).
} 
Key assumptions underlying TPR of close ties methods

In order for TPR of close ties to accurately estimate the incidence of abortion, the method must produce a robust estimation of the behavior of interest (the numerator) and a valid surrogate sample that is representative of the general population (the denominator). To accomplish this, six key assumptions inherent in these methods must be met (Table 1). While the assumptions listed below are described in language specific to the Confidante Method and abortion measurement, similar assumptions underlie all TPR of close ties methods to measure sensitive behaviors.

Measuring the numerator: creating an accurate indicator of abortions among confidantes

\section{Assumption 1. Respondents and confidantes share information about their abortions. All TPR} approaches require that information about the behavior in question is shared between social network ties. Prior research has suggested that the highly restrictive nature of abortion in many settings necessitates the sharing of abortion information; respondents and confidantes use their social networks for assistance in accessing abortion services, as these services are often not publicly advertised and may rely on referrals from trusted sources (Rossier, 2010; Rossier et al., 2006, 2018; Sedgh et al., 2011). Some have suggested that in settings where abortion is highly stigmatized yet broadly accessible (e.g., Ethiopia or India) there is less need for individuals to share information about their abortions with social network members (Rossier et al., 2018). However, individuals may still share their abortion experiences with close ties for other reasons (emotional support, shared experiences, etc.)

Assumption 2. Respondents will have complete knowledge of their confidantes' abortions. It is not sufficient for respondents and confidantes to generally share information about their abortions; respondents must additionally be aware of all abortions that have occurred among their listed confidantes. However, given the highly stigmatized nature of abortion in many settings, this assumption will almost certainly be violated, leading to a phenomenon known as transmission bias (Bernard et al., 2010; Killworth et al., 2006; Maltiel et al., 2015; Salganik et al., 2011). Transmission bias, or incomplete knowledge of abortions within social networks, has been identified as a major limitation in previous ATPR studies (Rossier, 2010). The Confidante Method was designed to reduce the impact of this bias by asking respondents to only think of the women with whom they share "intimate secrets", thereby increasing the likelihood that respondents will have better knowledge of their confidante's abortions. However, this specification is not sufficient to ensure that respondents have perfect knowledge of their 
confidantes' abortions, and it is likely that incidence estimates will be biased towards the null (as overreporting of abortion in unlikely). Any application of this method must therefore make some adjustment to account for transmission bias.

\section{Assumption 3. Respondents are willing and able to disclose information on confidantes' abortions}

in a survey. Irrespective of the completeness of respondent's information on confidante abortions, respondents must be willing to report this information in a survey. Social desirability bias may lead to violations of this assumption, which is a common problem when measuring sensitive behaviors (Tourangeau \& Yan, 2007). While it is well-established that respondents tend to under-report their own experiences of abortion (Jones and Forrest 1992; Jones and Kost 2007; Lindberg et al. 2020), the central assumption of the Confidante Method is that social desirability bias is lower when respondents report the abortions of other people rather than their own. While there is some evidence to support this for other sensitive behaviors (Bauman \& Fisher, 1986; Fisher, 1993), it is unclear how far these findings can be extrapolated to abortion.

Further, many applications of the Confidante Method aim to measure a time-defined incidence of abortion. Therefore, we must also assume that respondents are able to report the date of each confidante abortion with relative accuracy. However, respondents may also have incomplete or imperfect knowledge of when abortions occurred, leading to recall bias. Similar recall biases for the reporting of age of living siblings and at death have been documented in the sibling method, downwardly biases mortality estimates (Masquelier et al., 2021). While impactful events may be better remembered, respondents may also incorrectly infer recency from the clarity of the memory (Loftus \& Marburger, 1983; Sudman \& Bradburn, 1973). If abortion reporting is subject to this type of telescoping, one-year incidence estimates calculated from reports for the past year could become inflated.

Measuring the denominator: producing a surrogate sample of confidantes that is representative of the general population

Assumption 4. Respondents select confidantes with homophily. Helleringer et al. (2019) identified structural assumptions about underlying social networks that are essential for TPR to produce unbiased estimates. One of these assumptions it that the surrogate sample must be representative of the population of interest, which is assumed to occur through homophily (i.e. individual's tendency to seek out and know others who are similar to themselves). A violation of this assumption will result in homophily bias and 
create a non-representative sample of confidantes (Helleringer et al., 2019; McPherson et al., 2001; Rossier, 2010; Rossier et al., 2006).

\section{Assumption 5. Respondents who report no confidantes do not differ systematically from}

respondents who report any confidantes. Some respondents may not have any social network members who fit the confidante tie definition and thus contribute zero confidantes to the surrogate sample. In order to produce a robust incidence estimate, one must assume that respondents with zero confidantes do not differ systematically in socio-demographic characteristics, social network attributes, or abortion experiences from respondents with any confidantes. Violations of this assumption may result in the creation of an unrepresentative surrogate sample. Further, violations to this assumption could potentially lead to a biased estimation of abortion incidence due to a phenomenon known as "barrier effects". Barrier effects are when groups of people have differential propensities to know people in different groups, i.e. individuals who have abortions may be more likely to know others who have abortions. In the case of the Confidante Method, if respondents who report any confidantes have a higher abortion incidence rate than their counterpart respondents with zero confidantes, their corresponding confidantes may also be more likely to have abortions than the general population, ultimately resulting in a confidante incidence rate that is an overestimate of the true rate.

\section{Assumption 6. Confidantes' inclusion in the surrogate sample is independent of their abortion}

status. We assume that respondents will only identify confidantes based on the social-network tie definition provided, regardless of whether or not the confidante had an abortion. If respondents deliberately include (or exclude) social network members who are known to have had an abortion, the resulting surrogate sample will be biased. Further, independent of a respondent's selection motivations, we assume that a confidante's likelihood of being included in the surrogate sample is not associated with their likelihood of having had an abortion. If some confidantes are more popular (and therefore reported by multiple respondents) and popularity is correlated with abortion, the estimates will suffer from "popularity bias" (Helleringer et al., 2019). In the case of the Confidante Method, if respondents inappropriately select confidantes based on their abortion status, or some confidantes are more likely to be selected multiple times and this likelihood is correlated with their likelihood of abortion, then the resulting abortion incidence estimate will be unduly biased.

\section{Methods}

Data sources and sample 
This study utilizes data from an abortion module that the Guttmacher Institute designed and added to the 2018 PMA female surveys in Uganda and Ethiopia, which was fielded in partnership with Makerere University School of Public Health, Addis Ababa University, and Johns Hopkins Bloomberg School of Public Health. Surveys were conducted face-to-face on Android smartphones using Open Data Kit software. Data collection occurred from April-May 2018 in Uganda and from June-July 2018 in Ethiopia. The sampling plan in both countries was designed to produce a nationally representative sample of women aged 15-49 and relied on a two-stage cluster sampling design. A more detailed explanation of the PMA survey methodology and sampling procedure is provided elsewhere (PMA, 2018; Zimmerman et al., 2017).

A total of 4,454 female respondents were sampled in Uganda and 7,690 in Ethiopia. After excluding women who did not consent to participate in the survey (Uganda=166, Ethiopia=144), half of the respondents were randomized to be administered the Confidante module (Uganda=2,089, Ethiopia=3,725). To identify confidantes, each respondent was asked to think of her female friends or relatives with whom she shares "intimate secrets" and who shares "intimate secrets" with her. There was no mention of secrets as related to sexual activity or reproductive health status, and we did not specifically mention abortion in the consent materials for the study. Eligible confidantes were limited to women with whom the respondent has been in contact in the past year, are between the ages of 15 and 49, and currently live in the country. Respondents were asked to report up to three women who fit this description, each of whom was identified to the interviewer using a fake name. This process resulted in the identification of 3,268 confidantes in Uganda and 4,612 confidantes in Ethiopia.

The Institutional Review Boards of the Guttmacher Institute, Johns Hopkins Bloomberg School of Public Health, Makerere University, and Addis Ababa University provided ethical approval for the study.

\section{Measures}

Abortion was measured among respondents and confidantes. For each confidante, respondents were asked "as far as you know, has [confidante fake name] ever done something that intentionally ended a pregnancy?”3 Response options included “yes, I'm certain”, “yes, I think so”, and “no”. Respondents who replied "yes, I think so" constituted less than $2 \%$ of the responses across all confidantes in both countries (Appendix A). Therefore, "certain" and "probable" responses were collapsed to identify abortions among

\footnotetext{
${ }^{3}$ Other studies using the Confidante Method have used more expansive definitions of abortion to include actions around period regulation (i.e. doing something to bring on menstruation at a time when the respondent thought she was pregnant). (Bell, Shankar, et al., 2020; Keogh et al., 2020; Stillman et al., 2020)
} 
confidantes. For each reported confidante abortion, respondents were asked for the year in which their most recent abortion occurred. In some cases, respondents did not know the year of their confidante's abortion (5\% of lifetime confidante abortions in Ethiopia, 8\% in Uganda, Appendix B). If the respondent was not sure, she could choose from the following categories: "less than 1 year ago", "1 to less than 3 years ago", "3 to less than 5 years ago", or "5 or more years ago". To measure self-reported abortion, respondents were asked if they have ever done anything to successfully end a pregnancy. Respondents who indicated "yes, I succeeded" were coded as ever having had an abortion. Respondents were then asked what month and year their most recent abortion occurred.

Socio-demographic characteristics, including age, educational attainment, and region, were also measured for both respondents and confidantes. Additional socio-demographic characteristics collected only for respondents included household wealth, urban/rural residence, marital status, and parity.

\section{Analysis Plan}

We calculated one-year respondent and confidante abortion incidence estimates within each country. For the respondents, the numerator includes all abortions that occurred in the 12 months prior to the date of the interview, and the denominator is the number of respondents in the analytic sample. For confidantes, we did not collect the month that the abortion occurred, only calendar year. Therefore, the numerator for the confidante incidence rates includes all confidante abortions that occurred in 2017 (the last completed calendar year). If a respondent did not know the calendar year of their confidante's abortion, we included abortions if the respondent reported that the abortion occurred "less than one year ago" (Ethiopia n=0; Uganda, $n=2$.) The denominator for the confidante incidence rate is the total number of confidantes. Both the respondent and confidante incidence rates are then multiplied by 1,000 to get the incidence per 1,000 women of reproductive age. ${ }^{4}$

In order to adjust for the likelihood that respondents do not have perfect knowledge of their confidantes' abortions (Assumption 2), we calculated a transmission bias adjustment factor. Respondents with any confidantes who also self-reported an abortion were asked whether they told each of their confidantes about their abortion. We then calculated the proportion of respondents who told Confidantes 1, 2, and 3 .

\footnotetext{
${ }^{4}$ In order to ensure that reporting reliability did not diminish with each subsequent confidante, we first computed one-year incidence estimates separately among Confidantes 1-3 (Appendix B). After determining that abortion incidence did not dramatically decline across confidantes, we pooled all confidantes together to compute final oneyear incidence estimates for the full confidante sample.
} 
This is done separately for each confidante to account for the likelihood that sharing is most common between the respondent and Confidante 1 . We then compute an adjustment factor for the confidante abortion estimates that is the inverse of this proportion. (For example, if $70 \%$ of respondents who selfreported an abortion told their Confidante 1 about this abortion, then the adjustment factor would be $1.0 / 0.7$ or 1.43 . Given that these relationships are meant to be reciprocal, we would interpret this to mean for every Confidante 1 abortion reported by a respondent, there is an additional 0.43 abortions that are not being reported.) We then apply the confidante-specific adjustment factors to inflate the reported abortions in the past year separately among Confidantes 1,2, and 3. To get the one-year transmission bias adjusted abortion incidence estimates among all confidantes, we first sum the inflated number of abortions by each confidante, then divide by the total number of confidantes, and multiply this estimate by 1,000 .

Respondent abortion incidence estimates were weighted using the PMA-generated individual sample weights. However, we found evidence that respondents likely did not select confidantes with homophily, in violation of Assumption 4, as there were statistically significant differences in age, education level, and region between respondents and confidantes in both countries (Appendix C). In an effort to partially account for this violation, we used multivariable logistic regression to create post-stratification weights so that the confidante samples are nationally representative based on available characteristics. It is important to note that this procedure only adjusts for differences in characteristics that were measured for the confidantes; the reweighted confidante samples may still differ from the underlying populations in other important, yet unmeasured, ways.

\section{Sensitivity Analyses}

In order to determine whether recall bias in reporting of confidante abortion timing was present (in violation of Assumption 3), we calculated three-year incidence rates using the same methodology for determining the one-year rates (36 months from interview date for respondent, abortions that occurred less than three years ago or in calendar years 2015, 2016, and 2017 for confidantes), and then divided these estimates by three to create annualized one-year abortion incidence estimates. We then compare the annualized estimate to the directly reported confidante past one-year incidence estimate.

While we began with a nationally representative sample of respondents, we also observed that not all respondents reported having at least one confidante. This effectively removes these respondents' social networks from the sample, increasing the likelihood that the confidante sample will be non-representative. In order to assess whether barrier effects related to women's experiences with abortion are present (in 
violation of Assumption 5), we estimated unadjusted odds ratios to determine whether there were key differences in respondent abortion rates and socio-demographic characteristics based on whether or not they reported any confidantes.

\section{Validity check}

To test the overall performance of the method, we estimated another reproductive health behavior: the prevalence of IUD or implant use among confidantes. We asked respondents to report their confidantes' current use of IUDs or implants and calculated a corresponding Confidante prevalence rate. Similar to the respondents who self-reported abortions, we asked current IUD/implant users whether they reported this use to each of their confidantes and used this information to additionally adjust the Confidante Method produced IUD/implant estimates for transmission bias. We then compare the unadjusted and adjusted IUD/implant estimates to the self-reported prevalence calculated from the PMA survey as well as to the most recent estimates from the Demographic and Health Survey (DHS) in Ethiopia and Uganda.

All analyses were performed using Stata version 16.0 (StataCorp LP, College Station, TX).

\section{Results}

Approximately $25 \%$ of respondents in Ethiopia and 19\% of respondents in Uganda reported having zero confidantes, and the average number of confidantes per respondent was 1.3 in Ethiopia and 1.7 in Uganda (Figure 1). Confidante age, educational attainment, and region were similar to their corresponding respondents in each country, as a result of the post-stratification weighing (Table 2).

Figure 2 displays the proportion of respondents who reported their abortions to their respective confidantes. Contrary to our initial hypothesis, reporting did not decline in a linear way from Confidante 1 to Confidante 3 in either country, and the proportion of respondents who reported their abortions to their confidantes ranged from $41.7 \%$ to $56.8 \%$. See Appendix D for the transmission bias calculations.

Figure 3 shows one-year abortion incidence rates among respondents and confidantes as well as the most recent AICM estimate in each country. In Ethiopia, the unadjusted confidante abortion rate was almost double that of the respondents, though confidence intervals for the two estimates overlap (Confidantes: 7.1 per 1,000, 95\% CI:4.6-11.0; Respondents: 4.3 per 1,000, 95\% CI:2.5-7.3). After adjusting for transmission bias, the confidante abortion rate increased to 18.6 per 1,000 (95\% CI: 12.0-28.8). Both the unadjusted and transmission bias adjusted confidante rates were considerably lower than the 2014 AICM 
rate for Ethiopia (28.0 per 1,000, UI:23.5-33.0) (Moore et al., 2016). In Uganda, the difference between respondent and unadjusted confidante incidence rates was much larger, with the confidante rate being almost five times larger than the respondent rate (Confidantes: 39.5 per 1,000, 95\% CI:31.7-49.1; Respondents: 8.6 per 1,000, 95\% CI:4.7-15.8). While the unadjusted confidante rate is almost identical to the 2013 Uganda AICM incidence rate (39.0 per 1,000, UI:24.0-55.0) (Prada et al., 2016), the transmission bias adjusted rate appears unrealistic high, at almost double that of the most recent AICM estimate (76.8 per 1,000, 95\% CI:61.7-95.4).

\section{Sensitivity Tests}

Table 3 displays three-year and annualized one-year confidante abortion incidence estimates. In Ethiopia, the reported one-year confidante estimate is similar to the annualized estimate (6.1 per 1,000, 95\% CI:4.78.0). In Uganda, the one-year confidante estimate is almost twice as large as the annualized one-year estimate (24.3 per 1,000, 95\% CI:21.0-28.2), suggesting that reporting errors may have occurred when respondents reported the timing of their confidantes' abortions.

In both countries, older age, lower levels of education, and having more children were significantly associated with reporting zero confidantes (Table 4). In Ethiopia, rural residence, lower household wealth, and being married was also associated with having no confidantes. There were no statistically significant differences in one-year or three-year self-reported abortions for respondents in Ethiopia based on whether the respondents reported any confidantes. In Uganda, self-reporting an abortion was negatively associated with reporting zero confidantes, although unadjusted ORs were not statistically significant. Although not statistically significant, the one-year incidence rate among respondents in Uganda reporting 1+ confidante was almost double that of respondents reporting zero confidantes ( 9.4 per 1,000 vs. 5.3 per 1,000, $\mathrm{p}=0.102$; results not shown). An even larger gap exists for the three-year incidence estimates (15.5 per $1,000$ vs. 6.3 per $1,000, \mathrm{p}=0.051)$.

\section{Validity check}

In Ethiopia, the most recent DHS estimate for current IUD/implant use is 7.2\% (95\% CI:6.4-8.1) (CSA and ICF 2016), and respondent reports from the 2018 PMA survey were nearly identical to the DHS (7.5\%, 95\% CI:6.2-9.0) (Figure 4). In contrast, unadjusted confidante IUD/implant use estimate in Ethiopia was more than double that of the DHS or direct report estimates (18.5\%, 95\% CI:16.0-21.4). We observed a similar pattern in Uganda; the 2016 DHS and 2018 PMA estimates for current IUD/implant use were similar to each other $(\mathrm{DHS}=5.8 \%, 95 \% \mathrm{CI}: 5.4-6.3$; PMA=7.5\%, 95\% CI:6.2-9.0) (UBOS \& ICF, 2018), and the unadjusted confidante IUD/implant estimate was substantially larger than either the 
DHS or PMA rate $(24.5 \%, 95 \%$ CI:20.5-29.1). IUD/implant use appears to be a more visible SRH behavior than abortion, with $54 \%-88 \%$ of respondents reporting their current IUD/implant use to their corresponding confidante (Figure 5). As such, adjusting for transmission bias only slightly elevated the confidante IUD/implant use estimates (Ethiopia: 22.1\%, 95\% CI:19.1-25.5; Uganda: 27.8\%, 95\% CI:23.3-33.0). (See Appendix D for IUD/implant transmission bias calculations.) However, our validity check illustrates that the Confidante Method dramatically overestimates the prevalence of IUD and implant use in both countries.

\section{Discussion}

The results of this study provide important insights into the use of the Confidante Method to measure abortion incidence in clandestine settings. One of the largest difficulties in assessing new methods for measuring abortion incidence is that there is no gold standard against which to compare the estimates to fully validate the results. However, the results from this study nevertheless suggest that the Confidante Method is not a solution to the challenges of abortion measurement. Several of the method's foundational assumptions were violated, and our analysis revealed important biases in both the numerator and denominator for producing the Confidante estimate, as well as variation in these biases between the two countries. Efforts to correct for violations in the key assumption either failed or only partially addressed the resulting biases. It is not surprising, then, that our external validity check revealed that the Confidante Method failed to estimate a valid IUD/implant prevalence rate in either country.

\section{Evaluation of assumptions associated with the numerator}

In both study settings, it appears that Assumption 1 (respondents and confidantes share information about their abortions), was not violated; it is clear that at least some transfer of abortion information is occurring, given the frequency and certainty with which respondents report confidante abortions. However, in line with our a priori hypothesis, our results suggest that Assumption 2 (respondents have complete knowledge of their confidantes' abortions) was violated. One indication of this is that respondents only disclosed their own abortions to approximately half of their confidantes. Unfortunately, there are several major methodological limitations to accurately measure the magnitude of this bias. When designing our method for measuring transmission bias, we hypothesized that most respondents learned of confidante abortions directly from their confidante, as the social tie definition stipulated that the respondent-confidante dyad reciprocally share intimate secrets. As such, we asked respondents if they told their confidante directly about their abortion. However, it may be that individuals learn of others' 
abortions indirectly through other social network members. Further, even if we had been able to modify our measure of transmission to capture these indirect instances of abortion information sharing, which may partially reduce potential measurement error, respondents could still be unaware of all instances of indirect knowledge transfer. If this type of indirect information sharing is common, it may have inappropriately inflated our transmission bias adjustment factors.

In addition, our measure of transmission bias likely suffered from selection bias; we were only able to measure abortion information transmission among women who disclosed their abortion in the survey. Women who self-report abortions in research settings may also be more or less likely to disclose their abortions to their close confidantes, which would introduce error into our measurement of transmission bias. Finally, there is no way to validate our method for measuring transmission bias or test for the magnitude of the concerns outlined above. Previous Confidante Method studies have used an alternative approach, where respondents' certainty about their confidantes' abortions is used as a proxy for transmission bias, arguing that the inclusion of "less certain" abortions accounts for the incomplete transmission of abortion information (Bell, Shankar, et al., 2020). However, this method will never appropriately adjust for transmission bias, as it cannot account for abortions for which the respondent is completely unaware. Future research is needed to better understand how abortion information is transmitted within social networks and how transmission varies across cultural contexts in order to develop more robust methods for measuring transmission bias among confidantes.

Although transmission bias was measured in a standardized way in both Ethiopia and Uganda, adjusting for this bias produced very different results in the two countries. In Uganda, the transmission bias adjusted abortion rate was improbably high. Conversely, the adjusted abortion rate in Ethiopia was lower than the AICM estimate, suggesting it was too low if we consider the AICM estimate to be the most valid measure of abortion with which to compare. These results highlight another concern with appropriately accounting for transmission bias: even if it is accurately measured, it requires that the unadjusted abortion incidence estimates are not unduly biased. In this study, we found that other foundational assumptions were indeed violated (described in detail below), resulting in the inaccurate measurement of the unadjusted abortion rates. In this case, even if transmission bias is accurately measured, adjusting incidence estimates for transmission bias will amplify other sources of bias.

Assumption 3 addresses respondents' willingness and ability to accurately disclose the confidantes' abortions of which they were aware. Our sensitivity test results from Uganda, which showed large differences between the unadjusted one-year and annualized one-year abortion incidence rates, suggest that respondents likely had difficulty accurately reporting abortion information. Similar results were 
found in a study in Ghana using the Confidante Method, where abortion rates in the past year were over twice as high as those estimated occurring 1-2 years ago (Keogh et al., 2020). This may be evidence of telescoping, with respondents inappropriately including some confidante abortions as occurring "in the past year". Further, a recent US-based study found that accurate abortion reporting varies by length of recall (Lindberg \& Scott, 2018), which could also explain the differences between one and three-year rates. A better understanding of the extent to which telescoping and recall bias influence the reporting of confidante abortion is needed.

While the Uganda results indicate that respondents may not be able to report confidante abortions with appropriate accuracy, the results from Ethiopia suggest that respondents may not be willing to report all available confidante abortion information. The directly-reported respondent abortion rate was improbably low, and the unadjusted confidante rate was only slightly higher. While this could suggest that respondents in Ethiopia were less aware of their confidante's abortions, the highly stigmatize nature of abortion in Ethiopia may have made confidante abortion reporting more sensitive to social desirability bias (Rossier et al., 2018). Recent work supports this notion; in Java, Indonesia and Rajasthan, India, confidante abortion rates were implausibly low when compared to other abortion estimates for those countries, which were both similarly attributed to respondents' willingness to report confidante abortions (Bell, Shankar, et al., 2020; Stillman et al., 2020).

Differences in abortion reporting in Uganda and Ethiopia highlight the role that cultural contexts likely play in third-party reporting. The violations described above point to the need for formative research to investigate the appropriateness and design of the Confidante Method in specific study settings before it is implemented widely. This formative research should examine the extent to which Assumptions 1, 2, and 3 may be violated, as well as provide culturally-specific insight into nuances in the language used to define a "confidante" or discuss abortion in a given setting. To date, many applications of the Confidante Method, including the ones described in this paper, have not conducted formative research specifically designed to answer these questions and concerns. This is a missed opportunity to evaluate the appropriateness of this method and/or improve its implementation in a particular setting.

\section{Evaluation of assumptions associated with the denominator}

This study found extensive violations to assumptions associated with the denominator, likely resulting in unrepresentative surrogate samples in both countries. First, when we compare the confidante sample to respondents on key socio-demographic characteristics, we found significant differences between the pooled confidante samples and the nationally representative sample of respondents, violating Assumption 
4. This finding is in contrast to prior work by Helleringer et al. (2019), which found that the surrogate sample of friends generated through TPR was comparable to the respondent sample. Several differences between our applications of Confidante Method and the Helleringer TPR study may explain the lack of homophily between respondents and confidantes in Uganda and Ethiopia, including the definition of the tie (both in terms of geographic spread and contact frequency) as well as the number of reported confidantes/social network members. Other Confidante studies have experienced similar issues violating the assumption of homophily, with systematic differences between nationally representative samples of respondents and their corresponding confidante samples observed in Cote D'Ivoire, Ghana, Indonesia, and Nigeria (Bell, Shankar, et al., 2020; Keogh et al., 2020; Stillman et al., 2020). While we partially addressed this violation by creating adjusted confidante weights, our confidante samples are likely still unrepresentative, as we could only adjust for a limited set of observed characteristics (e.g., region, education, and age). Collecting more data on confidante characteristics could further improve the weighting adjustment. However, if the surrogate and respondent samples differ systematically by unmeasured or unobservable characteristics, this approach may never adequately address the issue of surrogate sample representativeness. If these differences are associated with the outcome of interest, it will ultimately result in a biased abortion incidence estimate. The underlying premise behind the confidante method is that it can produce representative estimates due to homophily; however, as this and several other studies have shown, the principle of homophily between respondents and confidantes is frequently violated. The continued violation of this foundational hypothesis to the confidante method raises serious questions about this method's ability to generate a representative surrogate sample. Future research using the Confidante Method must investigate whether changes to the tie definition or number of reported confidantes is an adequate solution to this major flaw before the method can be used to generate accurate incidence estimates.

Another cause of the unrepresentativeness of the surrogate confidante sample are systematic differences between respondents who report zero versus any confidantes; respondents with no confidantes were more likely to be married, have children, be less educated, and live in a household with less wealth than respondents with at least one confidante. This suggests that "zero confidante" respondents are more socially marginalized, and therefore a similar group of women is likely "missing" from the confidante sample. This is a major limitation of the Confidante Method in measuring abortion incidence; socially isolated and/or marginalized women will likely always be underrepresented due to the social connectedness necessary to be included in the confidante sample. These women may have different abortion experiences, and thus their exclusion would bias results. 
Biases introduced by respondents reporting zero confidantes have been documented in other social network-based incidence studies, including the problem of the "zero female respondent" in the Sibling Method (Gakidou \& King, 2006; Masquelier, 2013). Gakidou and King (2006) proposed a weighting adjustment to correct for this problem in measuring mortality, which has been adapted by other Confidante Method studies (Bell, Omoluabi, et al., 2020; Bell, Sheehy, et al., 2020; Keogh et al., 2020). The adjustment for "missing" confidantes is intended to address the problem that respondents who report zero confidantes are not represented in the surrogate sample. In this approach, "missing" confidantes are added to the surrogate sample by assuming that each respondent who reported zero confidantes has one confidante who shared their characteristics (i.e., assuming perfect homophily). Next, the relationships between reported confidante abortions and other respondent and confidante characteristics are used to predict the probability that each "missing" confidante had an abortion (Bell, Shankar, et al., 2020).

While the issue of "missing" confidantes certainly biased the abortion incidence estimates produced in this study, we did not attempt to adjust for this bias using a similar approach, as we find that adjustments to the sample in this way likely introduce a number of additional biases of unknown magnitude. First, by assigning the "missing" confidantes the characteristics of the corresponding respondent, one must assume perfect homophily between the respondents and this group of confidantes. However, in both Ethiopia and Uganda, we did not observe homophily between respondents and confidantes, and as such, it is not defensible to assume that there was homophily between respondents who reported zero confidantes and an imputed confidante. Further, this approach assumes that the relationship between reported confidante abortions and corresponding respondent/confidante characteristics will be similar for the missing group of socially-marginalized confidantes. However, the very nature of their marginalization may also make them more or less likely to have had an abortion, potentially invalidating this assumption. Finally, in both Uganda and Ethiopia, a sizeable proportion of respondents reported zero confidantes, meaning that using this approach would have amounted to imputing approximately $20 \%$ of the data used to estimate abortion incidence. Given the fact that this imputation approach requires two substantial assumptions that are not supported by the available data, it seemed untenable to produce estimates when a large portion of the data was imputed and not observed. Other studies using this adjustment technique have imputed even larger proportions of their data, ranging from one-third in Ghana (Keogh et al., 2020) to over 50\% in Nigeria (Bell, Omoluabi, et al., 2020; Bell, Sheehy, et al., 2020), and interpretations of the resulting incidence estimates should be made with caution.

It is difficult to tell whether Assumption 6 (respondents will select confidantes independently of their abortion status) was held or violated. A recent Confidante Method study in Ghana found that this 
assumption was likely violated, which may have inadvertently inflated the resulting abortion incidence estimate (Keogh et al., 2020). In that study, the researchers theorize respondents were primed to think of abortion while they were selecting their confidantes, which possibly lead respondents to purposely select confidantes who had previous abortion experiences. While it is difficult to assess whether this assumption was violated in our current study, our study design likely reduced the probability that this occurred; we did not mention abortion previously in the survey, and we did not mention sexual health in the description of the kinds of information confidantes should share with each other. However, we do not know how respondents may have interpreted the phrase "intimate secrets"; some respondents may have interpreted this to mean secrets related to sexual or reproductive matters, including abortion. Further, the logic underpinning the Confidante Method suggests that stigmatized and sensitive personal information is only disclosed between close social ties. However, it is possible that, the very act of sharing abortion information may cause individuals to consider each other "confidantes" according to the study definition of sharing "intimate secrets" who may not have otherwise considered the relationship to be a close tie. The extent to which this may be occurring represents a violation of Assumption 6.

In light of the extensive violations to the assumptions associated with the denominator, it may not be surprising that our validation check failed, with the Confidante Method resulting in a gross over-estimate of IUD and implant use. Given that abortion is chronically under-reported, there is a pervasive assumption in the field of abortion measurement that the highest estimate in a given setting is likely the least biased. However, our validation check suggests that the concerns with measuring the denominator may lead the Confidante Method to over-estimate abortion incidence. We strongly caution against the interpretation of a Confidante Method-produced abortion incidence estimate as the most accurate solely based on its greater magnitude.

Despite the many weaknesses outlined above of the Confidante Method, one positive feature of the method is that it allows for the collection of background characteristics of members of the hidden population, as well as additional information related to the sensitive behavior under study. For example, applications of the Confidante Method have contributed valuable insight into the safety of induced abortion in Nigeria, Cote d'Ivoire, and Rajasthan, India (Bell et al., 2019). Even though the safety estimates derived from this method are likely biased, this work is still essential to the design of policies and programs for addressing the needs of individuals seeking abortion in these countries. This benefit differentiates the method from other indirect methods for measuring abortion, such as the Network ScaleUp Method, which can only produce an overall incidence estimate of incidence (Sully et al., 2020). This presents an opportunity for further methodological improvements; the greater extent to which biases 
associated with the Confidante Method can be quantified and adjusted for, the more readily this method can be used to understand sociodemographic differences in risk factors associated with sensitive behaviors.

\section{Conclusions}

The Confidante Method is likely unsuitable for the accurate measurement of abortion incidence. Our anayses revealed several major limitations as well as pervasive violations to key assumptions. Some of this failure may have to do with the behavior in question; abortion is notoriously challenging to measure, and it may be particularly sensitive to the biases we observed in measuring the numerator. Given the variability in abortion stigma across setting, violations of key Confidante Method assumptions may be particularly sensitive to the cultural context where the study is implemented. To address these concerns, it is imperative that future studies first conduct formative research to understand the appropriateness of using the Confidante Method to measure abortion, as well as how the method should be implemented. This formative research should investigate several facets, including levels of abortion-related stigma in the study-specific context, how information is shared within social networks to better estimate the visibility of abortion, the most appropriate definition of a social tie to elicit accurate reporting, the cultural and social norms around disclosing abortion information, and the types of details that respondents might be able to report on about their confidantes' abortions.

Our results also revealed larger problems with the basic structural assumptions associated with the creation the surrogate sample. Violations to assumptions associated with the denominator are pervasive in this study, exist regardless of cultural context, and are more difficult to address with adjustments. This finding is more problematic than the biases observed in the numerator, as it has implications beyond the use of the Confidante Method to measure abortion and extends to other TPR approaches for measuring hidden or stigmatized behaviors. Major concerns shared across these approaches are the sensitivity of estimates to social-tie definitions and violations to the assumption of homophily. For example, the success of the Sibling Method may stem from the clearly defined nature of the sibling tie, while the Confidante Method definition of "secret sharing" was likely too interpretive, resulting in an inappropriate surrogate sample more prone to biases. Future research is needed to thoroughly investigate the extent to which differences in key features that distinguish TPR methods, including definitions of social tie relationships and numbers of social network members selected, result in unduly biased estimates of sensitive behaviors. In addition, it is important for future research using TPR approaches include external validation tests in order to better evaluate the robustness of the resulting estimates. Understanding the 
magnitude and direction of biases associated with the TPR is essential before the generated estimates can provide insight into the incidence of hidden or sensitive behaviors. 


\section{References}

Bauman, K. E., \& Fisher, L. A. (1986). On the measurement of friend behavior in research on friend influence and selection: Findings from longitudinal studies of adolescent smoking and drinking. Journal of Youth and Adolescence, 15(4), 345-353. https://doi.org/10.1007/BF02145731

Bell, S. O., OlaOlorun, F., Shankar, M., Ahmad, D., Guiella, G., Omoluabi, E., Khanna, A., Hyacinthe, A. K., \& Moreau, C. (2019). Measurement of abortion safety using community-based surveys: Findings from three countries. PLOS ONE, 14(11), e0223146. https://doi.org/10.1371/journal.pone.0223146

Bell, S. O., Omoluabi, E., OlaOlorun, F., Shankar, M., \& Moreau, C. (2020). Inequities in the incidence and safety of abortion in Nigeria. BMJ Global Health, 5(1), e001814. https://doi.org/10.1136/bmjgh-2019-001814

Bell, S. O., Shankar, M., Omoluabi, E., Khanna, A., Andoh, H. K., OlaOlorun, F., Ahmad, D., Guiella, G., Ahmed, S., \& Moreau, C. (2020). Social network-based measurement of abortion incidence: Promising findings from population-based surveys in Nigeria, Cote d'Ivoire, and Rajasthan, India. Population Health Metrics, 18(1), 28. https://doi.org/10.1186/s12963-020-00235-y

Bell, S. O., Sheehy, G., Hyacinthe, A. K., Guiella, G., \& Moreau, C. (2020). Induced abortion incidence and safety in Côte d'Ivoire. PLOS ONE, 15(5), e0232364. https://doi.org/10.1371/journal.pone.0232364

Bernard, H. R., Hallett, T., Iovita, A., Johnsen, E. C., Lyerla, R., McCarty, C., Mahy, M., Salganik, M. J., Saliuk, T., Scutelniciuc, O., Shelley, G. A., Sirinirund, P., Weir, S., \& Stroup, D. F. (2010). Counting hard-to-count populations: The network scale-up method for public health. Sexually Transmitted Infections, 86(Suppl_2), ii11-ii15. https://doi.org/10.1136/sti.2010.044446

Bicego, G. (1997). Estimating adult mortality rates in the context of the AIDS epidemic in sub-Saharan Africa: Analysis of DHS sibling histories. Health Transition Review, 7, 7-22.

CSA [Ethiopia], \& ICF. (2016). Ethiopia Demographic and Health Survey 2016 (p. 113). CSA and ICF. 
Fisher, R. J. (1993). Social Desirability Bias and the Validity of Indirect Questioning. Journal of Consumer Research, 20(2), 303-315. https://doi.org/10.1086/209351

Gakidou, E., \& King, G. (2006). Death by survey: Estimating adult mortality without selection bias from sibling survival data. Demography, 43(3), 569-585. https://doi.org/10.1353/dem.2006.0024

Graham, W., Brass, W., \& Snow, R. W. (1989). Estimating maternal mortality: The sisterhood method. Studies in Family Planning, 20(3), 125-135.

Helleringer, S., Adams, J., Yeatman, S., \& Mkandawire, J. (2019). Evaluating sampling biases from thirdparty reporting as a method for improving survey measures of sensitive behaviors. Social Networks, 59, 134-140. https://doi.org/10.1016/j.socnet.2019.07.003

Helleringer, S., Pison, G., Kanté, A. M., Duthé, G., \& Andro, A. (2014). Reporting Errors in Siblings’ Survival Histories and Their Impact on Adult Mortality Estimates: Results From a Record Linkage Study in Senegal. Demography, 51(2), 387-411. https://doi.org/10.1007/s13524-0130268-3

Hill, K., \& Trussell, J. (1977). Further developments in indirect mortality estimation. Population Studies, 31(2), 313-334. https://doi.org/10.1080/00324728.1977.10410432

Ishoso, D. K., Tshefu, A. K., Delvaux, T., \& Coppieters, Y. (2019). Extent of induced abortions and occurrence of complications in Kinshasa, Democratic Republic of the Congo. Reproductive Health, 16(1), 49. https://doi.org/10.1186/s12978-019-0727-4

Jones, E. F., \& Forrest, J. D. (1992). Underreporting of abortion in surveys of U.S. women: 1976 to 1988. Demography, 29(1), 113-126.

Jones, R. K., \& Kost, K. (2007). Underreporting of Induced and Spontaneous Abortion in the United States: An Analysis of the 2002 National Survey of Family Growth. Studies in Family Planning, 38(3), 187-197. https://doi.org/10.1111/j.1728-4465.2007.00130.x

Juarez, F., Cabigon, J., \& Singh, S. (2010). The Sealed Envelope Method of Estimating Induced Abortion: How Much of an Improvement? In S. Singh, L. Remez, \& A. Tartaglione (Eds.), 
Methodologies for Estimating Abortion Incidence and Abortion-Related Morbidity: A Review (pp. 107-124). Guttmacher Institute and International Union for the Scientific Study of Population.

Keogh, S. C., Otupiri, E., Chiu, D. W., Polis, C. B., Hussain, R., Bell, S. O., Nakua, E. K., \& LarsenReindorf, R. (2020). Estimating the incidence of abortion: A comparison of five approaches in Ghana. BMJ Global Health, 5(4), e002129. https://doi.org/10.1136/bmjgh-2019-002129

Killworth, P. D., McCarty, C., Johnsen, E. C., Bernard, H., \& Shelley, G. A. (2006). Investigating the variation of personal network size under unknown error conditions. Sociological Methods and Research, 35(1), 84-112. https://doi.org/10.1177/0049124106289160

Lindberg, L., Kost, K., Maddow-Zimet, I., Desai, S., \& Zolna, M. (2020). Abortion Reporting in the United States: An Assessment of Three National Fertility Surveys. Demography, 57(3), 899-925. https://doi.org/10.1007/s13524-020-00886-4

Lindberg, L., \& Scott, R. H. (2018). Effect of ACASI on Reporting of Abortion and Other Pregnancy Outcomes in the US National Survey of Family Growth. Studies in Family Planning, 49(3), 259278. https://doi.org/10.1111/sifp.12068

Loftus, E. F., \& Marburger, W. (1983). Since the eruption of Mt. St. Helens, has anyone beaten you up? Improving the accuracy of retrospective reports with landmarkevents. Memory \& Cognition, 11(2), 114-120. https://doi.org/10.3758/BF03213465

Maltiel, R., Raftery, A. E., McCormick, T. H., \& Baraff, A. J. (2015). Estimating Population Size Using the Network Scale Up Method. The Annals of Applied Statistics, 9(3), 1247-1277. https://doi.org/10.1214/15-AOAS827

Masquelier, B. (2013). Adult Mortality From Sibling Survival Data: A Reappraisal of Selection Biases. Demography, 50(1), 207-228. https://doi.org/10.1007/s13524-012-0149-1

Masquelier, B., Kanyangarara, M., Pison, G., Kanté, A. M., Ndiaye, C. T., Douillot, L., Duthé, G., Sokhna, C., Delaunay, V., \& Helleringer, S. (2021). Errors in reported ages and dates in surveys of adult mortality: A record linkage study in Niakhar (Senegal). Population Studies, O(0), 1-19. https://doi.org/10.1080/00324728.2020.1854332 
McPherson, M., Smith-Lovin, L., \& M Cook, J. (2001). Birds of a Feather: Homophily in Social Networks. Аnnu Rev Sociol, 27, 415-444. https://doi.org/10.1146/annurev.soc.27.1.415

Moore, A., Gebrehiwot, Y., Fetters, T., Wado, Y., Bankole, A., Singh, S., Gebreselassie, H., \& Getachew, Y. (2016). The Estimated Incidence of Induced Abortion in Ethiopia, 2014: Changes in the Provision of Services Since 2008. International Perspectives on Sexual and Reproductive Health, 42(3), 111-120. https://doi.org/10.1363/42e1816

Moseson, H., Treleaven, E., Gerdts, C., \& Diamond-Smith, N. (2017). The List Experiment for Measuring Abortion: What We Know and What We Need. Studies in Family Planning, 48(4), 397-405. https://doi.org/10.1111/sifp.12042

Obermeyer, Z., Rajaratnam, J. K., Park, C. H., Gakidou, E., Hogan, M. C., Lopez, A. D., \& Murray, C. J. L. (2010). Measuring Adult Mortality Using Sibling Survival: A New Analytical Method and New Results for 44 Countries, 1974-2006. PLOS Medicine, 7(4), e1000260. https://doi.org/10.1371/journal.pmed.1000260

Oliveras, E., \& Letamo, G. (2010). Examples of Methods to Address Underreporting of Induced Abortion: Preceding Birth Interval Technique and Randomized Response Technique. In S. Singh, Lisa Remez, \& Alyssa Tartaglione (Eds.), Methodologies for Estimating Abortion Incidence and Abortion-Related Morbidity: A Review (pp. 63-70). Guttmacher Institute and International Union for the Scientific Study of Population.

PMA. (2018). Survey Methodology | PMA2020. Performance Monitoring and Accountability 2020. https://www.pma2020.org/survey-methodology

Prada, E., Atuyambe, L. M., Blades, N. M., Bukenya, J. N., Orach, C. G., \& Bankole, A. (2016). Incidence of Induced Abortion in Uganda, 2013: New Estimates Since 2003. PLOS ONE, 11(11), e0165812. https://doi.org/10.1371/journal.pone.0165812

Rossier, C. (2003). Estimating Induced Abortion Rates: A Review. Studies in Family Planning, 34(2), 87-102. https://doi.org/10.1111/j.1728-4465.2003.00087.x 
Rossier, C. (2010). Measuring Abortion with the Anonymous Third Party Reporting Method. In S. Singh, L. Remez, \& A. Tartaglione (Eds.), Methodologies for Estimating Abortion Incidence and Abortion-Related Morbidity: A Review. Guttmacher Institute and International Union for the Scientific Study of Population.

Rossier, C., Feehan, D., Olowabi, O., Marchin, A., \& Kim, C. (2018, December). A multiplex RDS for abortion? Assessing the potential of the Respondent-Driven Sampling to study abortion safety in restrictive contexts. International Union for the Scientific Study of Population, Watamu, Kenya.

Rossier, C., Guiella, G., Ouédraogo, A., \& Thiéba, B. (2006). Estimating clandestine abortion with the confidants method-Results from Ouagadougou, Burkina Faso. Social Science \& Medicine, 62(1), 254-266. https://doi.org/10.1016/j.socscimed.2005.05.024

Rutenberg, N., \& Sullivan, J. M. (1991). Direct and Indirect Estimates of Maternal Mortality From the Sisterhood Method (No. 3; Demographic and Health Surveys World Conference Proceedings, pp. 1669-1696). https://www.semanticscholar.org/paper/Direct-and-Indirect-Estimates-of-MaternalMortality-Rutenberg-Sullivan/23882db29007a6aa976a56a2af7009acb9833694

Salganik, M., Mello, M., Abdo, A., Bertoni, N., Fazito, D., \& Bastos, F. (2011). The Game of Contacts: Estimating the Social Visibility of Groups. Social Networks, 33(1), 70-78. https://doi.org/10.1016/j.socnet.2010.10.006

Sedgh, G., \& Keogh, S. C. (2019). Novel approaches to estimating abortion incidence. Reproductive Health, 16(1), 44. https://doi.org/10.1186/s12978-019-0702-0

Sedgh, G., Rossier, C., Kabore, I., Bankole, A., \& Mikulich, M. (2011). Estimating Abortion Incidence in Burkina Faso Using Two Methodologies. Studies in Family Planning, 42, 147-154.

Singh, S., Prada, E., \& Juarez, F. (2010). The Abortion Incidence Complications Method: A Quantitative Technique. In S. Singh, L. Remez, \& A. Tartaglione (Eds.), Methodologies for Estimating Abortion Incidence and Abortion-Related Morbidity: A Review (pp. 71-98). Guttmacher Institute and International Union for the Scientific Study of Population. 
Singh, S., Remez, L., Sedgh, G., Kwok, L., \& Onda, T. (2018). Abortion Worldwide 2017: Uneven Progress and Unequal Access. Guttmacher Institute. https://www.guttmacher.org/sites/default/files/report_pdf/abortion-worldwide-2017.pdf

Stanton, C., Abderrahim, N., \& Hill, K. (2000). An assessment of DHS maternal mortality indicators. Studies in Family Planning, 31(2), 111-123. https://doi.org/10.1111/j.1728-4465.2000.00111.x

Stillman, M., Leong, E., Utomo, B., Dadun, D., Aryanty, R. I., Sedgh, G., \& Giorgio, M. M. (2020). An Application of the Confidante Method to Estimate Induced Abortion Incidence in Java, Indonesia. International Perspectives on Sexual and Reproductive Health, 46, 199-210. https://doi.org/10.1363/46e0120

Sudman, S., \& Bradburn, N. M. (1973). Effects of Time and Memory Factors on Response in Surveys. Journal of the American Statistical Association, 68(344), 805-815. https://doi.org/10.1080/01621459.1973.10481428

Sully, E., Giorgio, M., \& Anjur-Dietrich, S. (2020). Estimating abortion incidence using the network scale-up method. Demographic Research, 43(56), 1651-1684. https://doi.org/10.4054/DemRes.2020.43.56

Timæus, I. M., \& Jasseh, M. (2004). Adult mortality in sub-Saharan Africa: Evidence from demographic and health surveys. Demography, 41(4), 757-772. https://doi.org/10.1353/dem.2004.0037

Tourangeau, R., \& Yan, T. (2007). Sensitive questions in surveys. Psychological Bulletin, 133(5), 859883. https://doi.org/10.1037/0033-2909.133.5.859

Trussell, J., \& Rodriguez, G. (1990). A Note on the Sisterhood Estimator of Maternal Mortality. Studies in Family Planning, 21(6), 344-346. JSTOR. https://doi.org/10.2307/1966923

UBOS \& ICF. (2018). Uganda Demographic and Health Survey 2016. UBOS and ICF.

Yeatman, S., \& Trinitapoli, J. (2011). Best-Friend Reports: A Tool for Measuring the Prevalence of Sensitive Behaviors. American Journal of Public Health, 101(9), 1666-1667. https://doi.org/10.2105/AJPH.2011.300194 
Zimmerman, L., Olson, H., PMA2020 Principal Investigators Group, Tsui, A., \& Radloff, S. (2017).

PMA2020: Rapid Turn-Around Survey Data to Monitor Family Planning Service and Practice in Ten Countries: PMA2020: Rapid Turn-Around Survey Data. Studies in Family Planning, 48(3), 293-303. https://doi.org/10.1111/sifp.12031 
Table 1. Assumptions of the Confidante Method

\begin{tabular}{|c|c|c|c|}
\hline & Assumption & $\begin{array}{l}\text { Biases created by } \\
\text { assumption } \\
\text { violations }\end{array}$ & Methods for identifying violations \\
\hline Assumption 1 & $\begin{array}{l}\text { Respondents and confidantes share } \\
\text { information about their abortions }\end{array}$ & Study design bias & $\begin{array}{l}\text { Investigate whether respondents report any abortions among } \\
\text { confidantes. } \\
\text { Ask respondents who self-report abortions whether they told } \\
\text { any of their confidantes about their own abortion. } \\
\text { Implausibly low confidante abortion rates may be evidence } \\
\text { that this assumption may have been violated. }\end{array}$ \\
\hline Assumption 2 & $\begin{array}{l}\text { Respondents will have complete } \\
\text { knowledge of their confidantes' } \\
\text { abortions }\end{array}$ & Transmission Bias & $\begin{array}{l}\circ \text { Assume this assumption is violated. } \\
\text { Confirm by asking respondents who self-report abortions } \\
\text { whether they shared this information with each of their } \\
\text { reported confidantes }\end{array}$ \\
\hline Assumption 3 & $\begin{array}{l}\text { Respondents are willing and able } \\
\text { to disclose information on } \\
\text { confidantes' abortions in a survey }\end{array}$ & $\begin{array}{l}\text { Social desirability bias } \\
\text { Recall bias }\end{array}$ & $\begin{array}{l}\text { Implausibly low confidante abortion rates are evidence that } \\
\text { this assumption may have been violated. } \\
\text { Compare respondents' direct reports of abortions to those of } \\
\text { abortions among confidantes. If the respondent abortion rate } \\
\text { is the same or higher than the confidante rate, then this } \\
\text { assumption has been violated. }\end{array}$ \\
\hline Assumption 4 & $\begin{array}{l}\text { Respondents select confidantes } \\
\text { with homophily }\end{array}$ & Selection bias & $\begin{array}{l}\text { Compare socio-demographic characteristics of the confidante } \\
\text { sample to the respondent sample or other nationally } \\
\text { representative sample. Systematic differences will indicate } \\
\text { that this assumption has been violated. }\end{array}$ \\
\hline Assumption 5 & $\begin{array}{l}\text { Respondents who report no } \\
\text { confidantes do not differ } \\
\text { systematically from respondents } \\
\text { who report any confidantes }\end{array}$ & $\begin{array}{l}\text { Selection bias } \\
\text { Barrier Effects }\end{array}$ & $\begin{array}{l}\text { Compare key characteristics between respondents who report } \\
\text { zero vs. any confidantes. Systematic differences between the } \\
\text { two samples will indicate that this assumption has been } \\
\text { violated. }\end{array}$ \\
\hline Assumption 6 & $\begin{array}{l}\text { Confidante inclusion in the } \\
\text { surrogate sample is independent of } \\
\text { their abortion status. }\end{array}$ & $\begin{array}{l}\text { Selection bias } \\
\text { Popularity bias }\end{array}$ & $\begin{array}{l}\text { Difficult to determine. } \\
\text { Investigate whether respondents were primed to think about } \\
\text { abortion prior to being asked to identify confidantes. An } \\
\text { overestimate of abortion incidence can also indicate that this } \\
\text { assumption was violated. }\end{array}$ \\
\hline
\end{tabular}


Table 2. Distribution of sociodemographic characteristics of respondents and confidantes in Ethiopia and Uganda

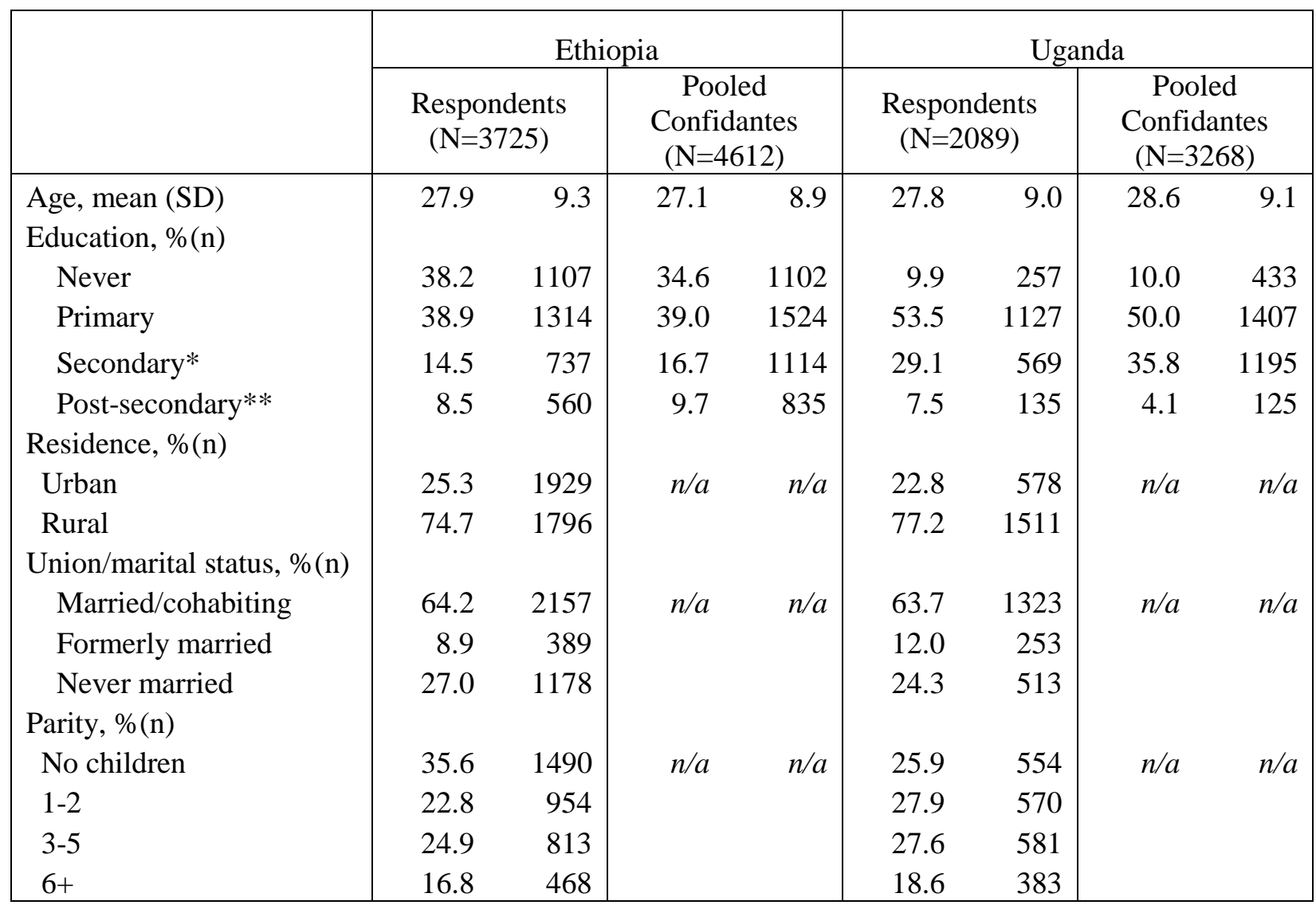

* Secondary includes: Secondary (ET); 'O' Level, 'A' Level (UG)

** Post-secondary includes: Technical, Higher (ET); Tertiary, University (UG)

Notes: Weighted proportions with unweighted Ns shown. Ns may not sum to total $\mathrm{N}$ due to missing data. Valid percentages shown.

Table 3. One-year, three-year, and annualized one-year confidante abortion incidence rates per 1,000 women aged 15-49 in Ethiopia and Uganda

\begin{tabular}{|l|c|c|c|c|c|c|}
\hline & \multicolumn{3}{|c|}{ Ethiopia } & \multicolumn{3}{c|}{ Uganda } \\
\hline & Rate & \multicolumn{2}{|c|}{$95 \%$ CIs } & Rate & \multicolumn{2}{c|}{$95 \%$ CIs } \\
\hline 1-year & 7.1 & 4.8 & 10.6 & 39.5 & 31.9 & 48.9 \\
\hline 3-year rate* & 18.4 & 14.2 & 23.9 & 73.0 & 62.9 & 84.5 \\
\hline Annualized 1-year & 6.1 & 4.7 & 8.0 & 24.3 & 21.0 & 28.2 \\
\hline
\end{tabular}

*Reflects confidante abortions reported to have occurred in 2015-2017 calendar years

Notes: Incidence estimates adjusted using post-stratification rates. Estimates have not been adjusted for transmission bias. 
Table 4. Unadjusted odds ratios for the relationship between respondent characteristics and reporting zero confidantes in Ethiopia and Uganda

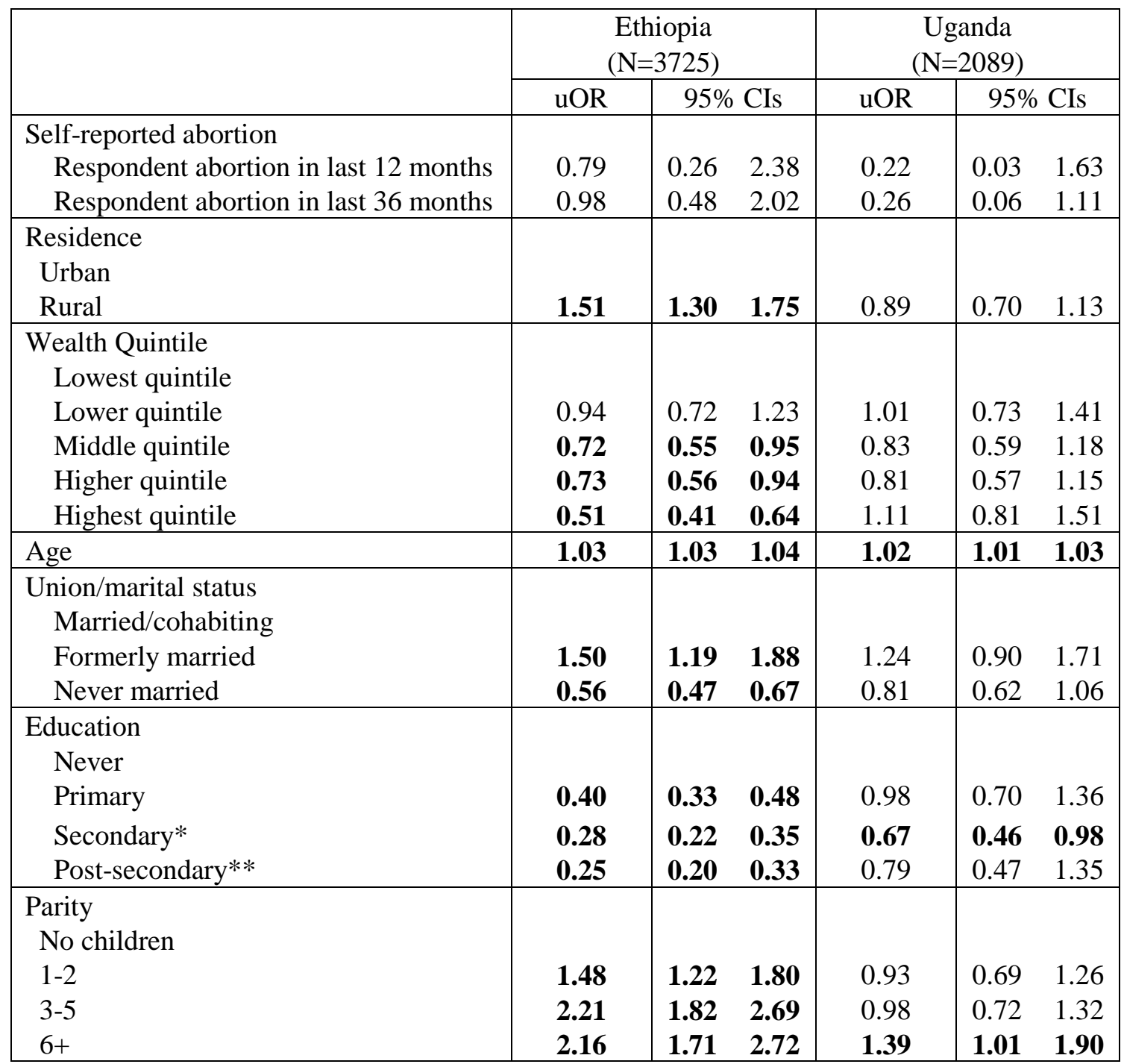

* Secondary includes: Secondary (ET); 'O' Level, 'A' Level (UG)

** Post-secondary includes: Technical, Higher (ET); Tertiary, University (UG)

Notes: Bolded results indicate statistical significance at $\mathrm{p}<0.05$ 


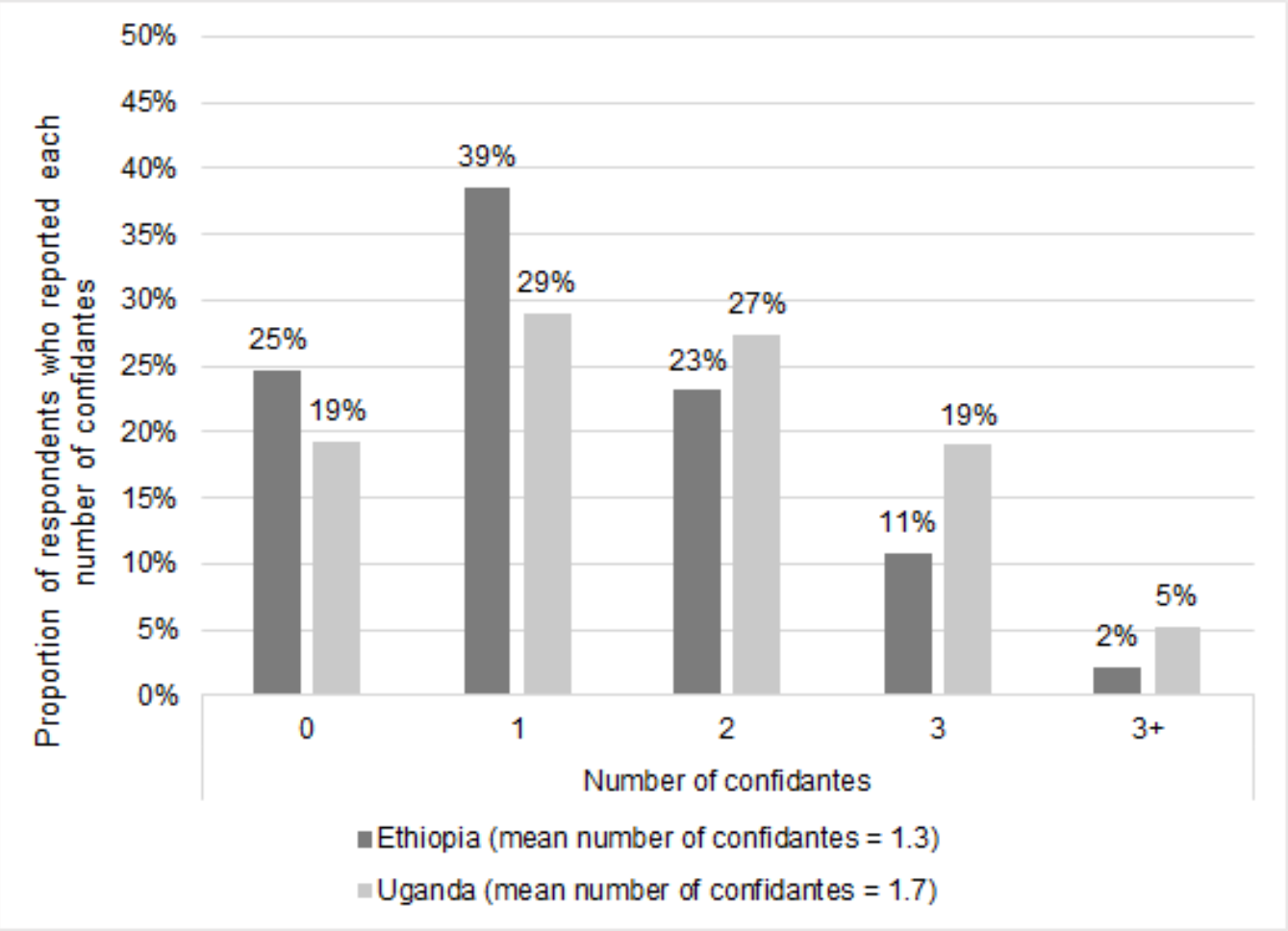

Figure 1. Number of confidantes reported by respondents in Ethiopia and Uganda 


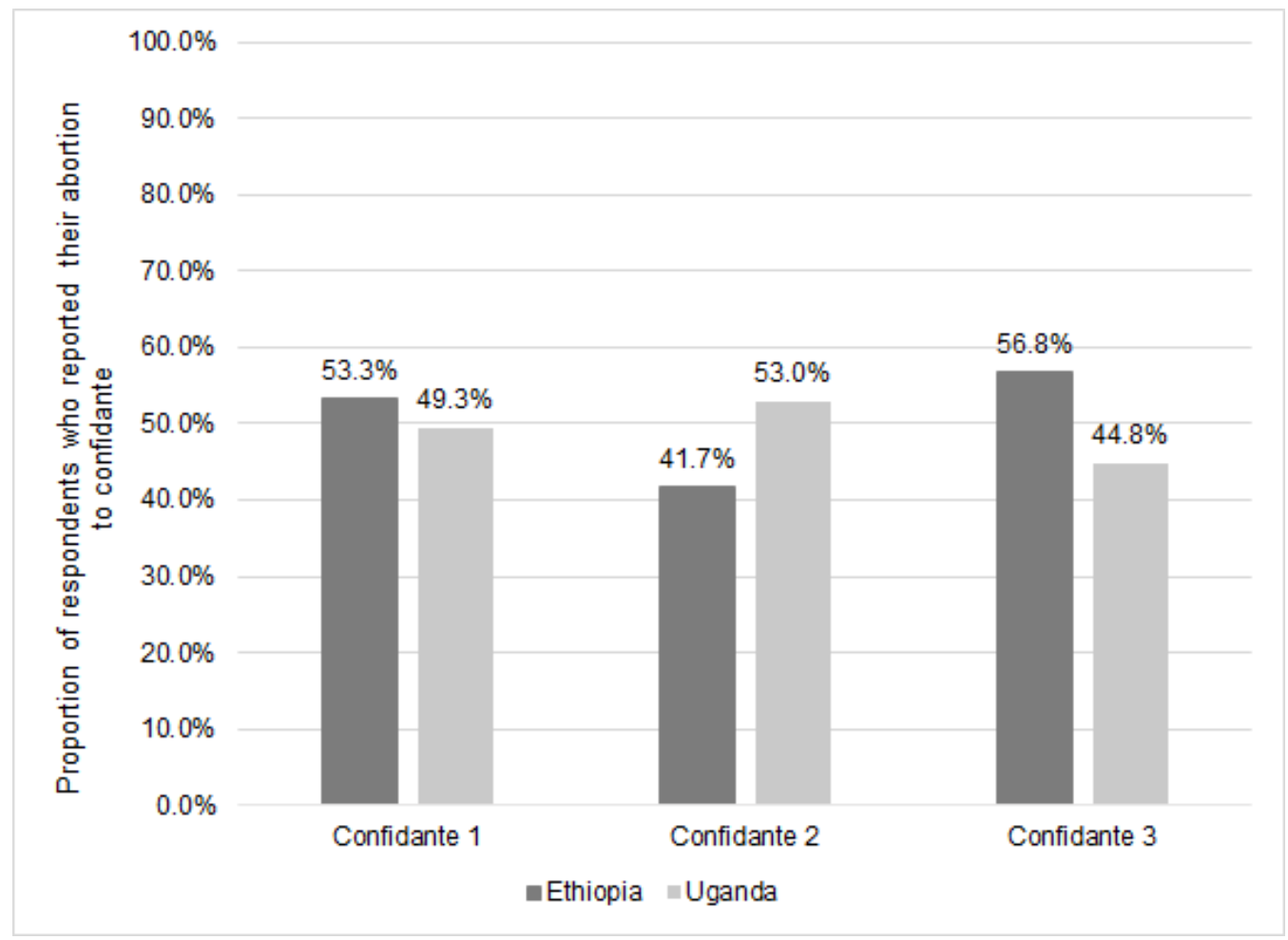

Figure 2. Proportion of respondents self-reporting abortion who told each confidante about their abortion in Ethiopia and Uganda 

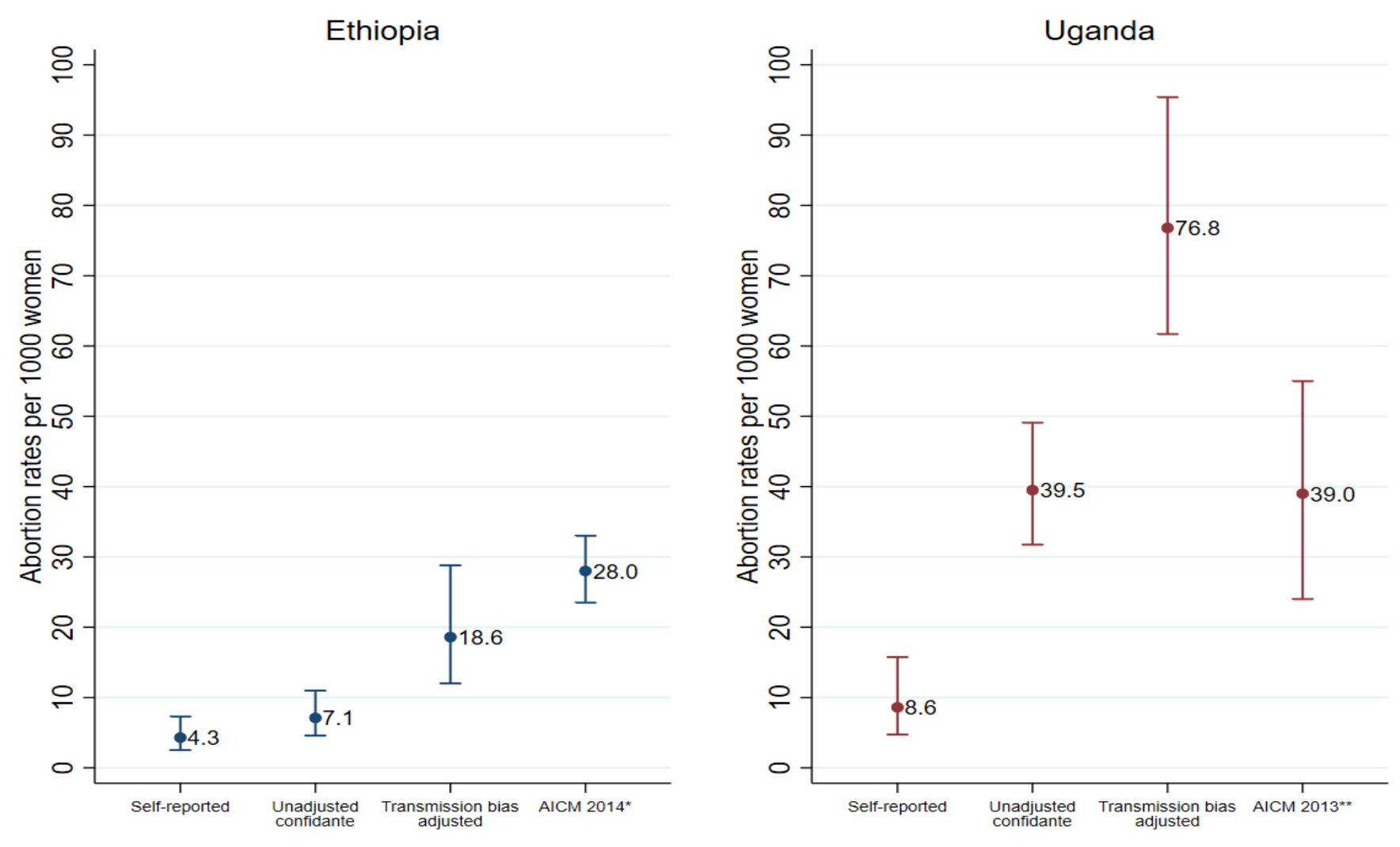

Figure 3. Self-reported, unadjusted confidante, transmission bias adjusted confidante, and AICM one-year abortion incidence rates per 1,000 women aged 15-49 in Ethiopia and Uganda

*Moore, A., Gebrehiwot, Y., Fetters, T., Wado, Y., Bankole, A., Singh, S., et al. (2016). The Estimated Incidence of Induced Abortion in Ethiopia, 2014: Changes in the Provision of Services Since 2008. International perspectives on sexual and reproductive health, 42(3), 111-120.

**Prada, E., Atuyambe, L. M., Blades, N. M., Bukenya, J. N., Orach, C. G., \& Bankole, A. (2016). Incidence of Induced Abortion in Uganda, 2013: New Estimates Since 2003. PLOS ONE, 11(11), e0165812. 

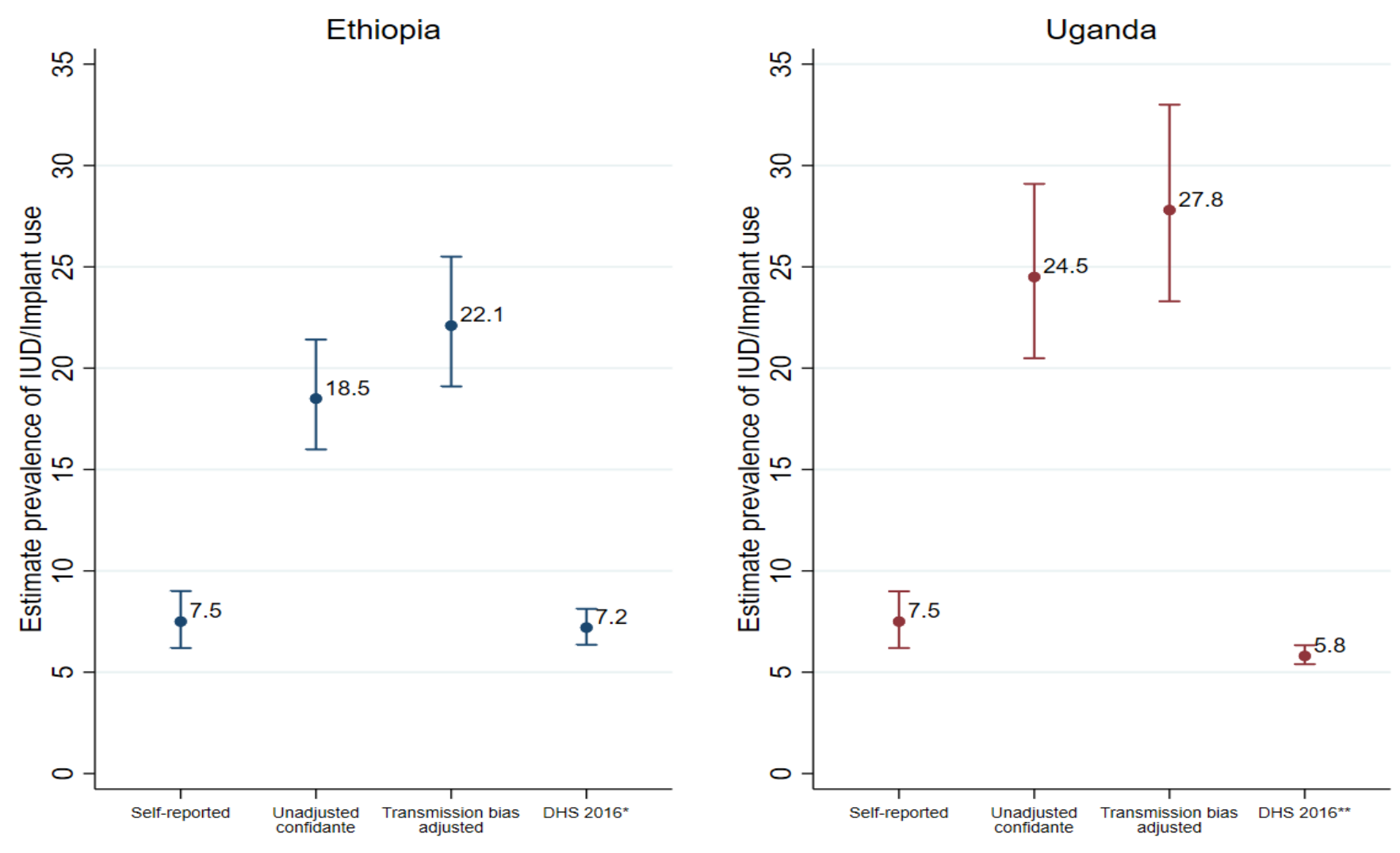

Figure 4. Self-reported, unadjusted confidante, transmission bias adjusted confidante, and DHS estimates of the current prevalence of intrauterine device and implant use in Ethiopia and Uganda

*Central Statistical Agency (CSA) [Ethiopia] and ICF. (2016). Ethiopia Demographic and Health Survey 2016 (p. 113). Addis Ababa, Ethiopia, and Rockville, Maryland, USA: CSA and ICF.

**UBOS \& ICF. (2018). Uganda Demographic and Health Survey 2016. Kampala, Uganda and Rockville, Maryland, USA: UBOS and ICF. 


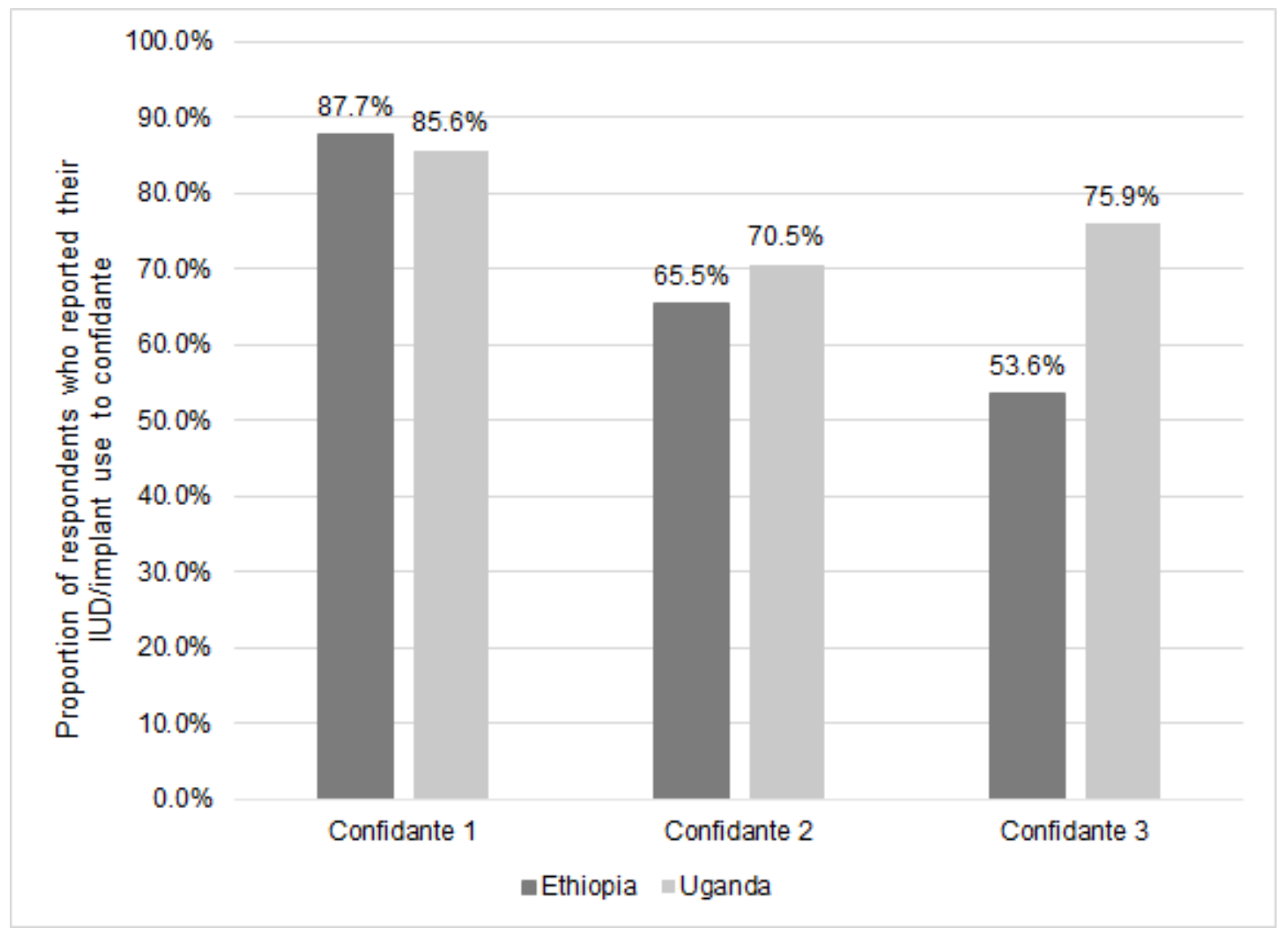

Figure 5. Proportion of respondents who reported their current intrauterine device or implant use to each confidante in Ethiopia and Uganda 


\section{Appendix A: Certainty in respondent reports of lifetime confidante abortions}

In both Uganda and Ethiopia, respondents displayed high levels of certainty in reporting that their confidantes had an abortion. Respondents reported uncertain lifetime abortions for less than $1 \%$ of confidantes in Ethiopia $(n=17)$ and 1.7\% $(n=54)$ in Uganda (Tables A1 and A2). In Ethiopia, this correspondents to approximately $93 \%$ of all reported confidante abortions $(n=217$ of 234) being reported by respondents with certainty. Similarly in Uganda, $88 \%$ ( $n=386$ of 440) were reported with certainty. These proportions remained relatively stable across confidantes.

Among reported lifetime confidante abortions, respondents were unable to report the year in which the abortion occurred in 5\% of cases $(n=11)$ in Ethiopia and $8 \%$ of cases $(n=34)$ in Uganda.

Table A1. Respondent reports of Confidante abortions in Ethiopia

\begin{tabular}{|c|c|c|c|c|}
\hline & $\begin{array}{c}\text { All } \\
\text { Confidantes } \\
(\mathrm{N}=4612)\end{array}$ & $\begin{array}{c}\text { Confidante } 1 \\
(\mathrm{~N}=2782)\end{array}$ & $\begin{array}{c}\text { Confidante } 2 \\
(\mathrm{~N}=1346)\end{array}$ & $\begin{array}{c}\text { Confidante } 3 \\
(\mathrm{~N}=484)\end{array}$ \\
\hline Confidante ever had an abortion, \% (n) & & & & \\
\hline Yes, I am certain & $4.7(217)$ & $5.1(142)$ & $4.5(60)$ & $3.1(15)$ \\
\hline Yes, I think so & $0.4(17)$ & $0.5(15)$ & $0.1(2)$ & $0(0)$ \\
\hline No & $94.9(4378)$ & $94.3(2625)$ & $95.4(1284)$ & $96.9(469)$ \\
\hline Reported confidante abortion & $(n=234)$ & $(n=157)$ & $(n=62)$ & $(n=15)$ \\
\hline $\begin{array}{l}\text { Respondent unable to report } \\
\text { abortion year, \% (n) }\end{array}$ & $4.74(11)$ & $5.7(9)$ & $1.6(1)$ & $6.7(1)$ \\
\hline
\end{tabular}

Table A2. Respondent reports of Confidante abortions in Uganda

\begin{tabular}{|c|c|c|c|c|}
\hline & $\begin{array}{c}\text { All } \\
\text { Confidantes } \\
(\mathrm{N}=3268)\end{array}$ & $\begin{array}{c}\text { Confidante } 1 \\
(\mathrm{~N}=1684)\end{array}$ & $\begin{array}{c}\text { Confidante } 2 \\
(\mathrm{~N}=1078)\end{array}$ & $\begin{array}{l}\text { Confidante } 3 \\
(\mathrm{~N}=506)\end{array}$ \\
\hline Confidante ever had an abortion, $\%$ (n) & & & & \\
\hline Yes, I am certain & $11.8(386)$ & $13.2(223)$ & $10.9(118)$ & $8.9(45)$ \\
\hline Yes, I think so & $1.7(54)$ & $1.7(28)$ & $1.6(17)$ & $1.8(9)$ \\
\hline No & $86.5(2828)$ & $85.1(1433)$ & $87.5(943)$ & $89.3(452)$ \\
\hline Reported confidante abortion & $(n=440)$ & $(n=251)$ & $(n=135)$ & $(n=54)$ \\
\hline $\begin{array}{r}\text { Respondent unable to report } \\
\text { abortion year, \% (n) }\end{array}$ & $7.7(34)$ & $9.6(24)$ & $5.9(8)$ & $3.7(2)$ \\
\hline
\end{tabular}




\section{Appendix B. Evidence that reporting reliability did not diminished dramatically with each subsequent confidante}

In order to ensure that reporting reliability did not diminish with each subsequent confidante, we computed one-year incidence estimates separately among Confidantes 1-3 (Table B1). The one-year abortion incidence rate in Ethiopia does not follow a clear pattern from Confidante 1 to 3. In Uganda, the abortion rate does drop after Confidante 1 (46.1\%) but levels out for Confidantes 2 or $3(33.1 \%, 31.0 \%)$. Given that we did not observe a steady decline in incidence estimates from Confidantes 1 to 3, analyses include all pooled confidantes to compute one-year incidence estimates for the full confidante sample.

Table B1. One-year abortion incidence estimates per 1,000 women aged 15-49, by confidante in Ethiopia and Uganda

\begin{tabular}{|c|c|c|c|}
\hline & $\begin{array}{c}\text { Abortion } \\
\text { Incidence Rate }\end{array}$ & \multicolumn{2}{c|}{ 95\% CI } \\
\hline & \multicolumn{3}{|c|}{ Ethiopia } \\
\hline Confidante 1 & 6.2 & 3.7 & 10.4 \\
\hline Confidante 2 & 9.9 & 5.2 & 18.8 \\
\hline Confidante 3 & 4.4 & 0.7 & 26.6 \\
\hline & 43.1 & 34.9 & 60.6 \\
\hline Confidante 1 & 33.1 & 21.6 & 50.4 \\
\hline Confidante 2 & 31.0 & 18.1 & 52.6 \\
\hline Confidante 3 & \multicolumn{3}{|c|}{ Uganda } \\
\hline
\end{tabular}

Notes: Incidence estimates adjusted using post-stratification rates. Estimates have not been adjusted for transmission bias. 


\section{Appendix C. Evidence that respondents likely did not select confidantes with homophily}

Tables $\mathrm{C} 1$ and $\mathrm{C} 2$ display evidence that respondents did not select confidantes with homophily, in violation of Assumption 4. There were statistically significant differences in age, education level, and region between respondents and confidantes in both Ethiopia and Uganda

Table C1. Differences in available sociodemographic characteristics between respondents and pooled confidantes in Ethiopia

\begin{tabular}{|c|c|c|c|c|c|}
\hline & \multicolumn{2}{|c|}{$\begin{array}{l}\text { Respondents } \\
(\mathrm{N}=3725)\end{array}$} & \multicolumn{2}{|c|}{$\begin{array}{l}\text { Pooled confidantes } \\
\quad(\mathrm{N}=4612)\end{array}$} & \multirow[t]{2}{*}{$\mathrm{p}$-value } \\
\hline & $\%$ & $\mathrm{~N}$ & $\%$ & $\mathrm{~N}$ & \\
\hline Age & & & & & $<0.001$ \\
\hline $15-19$ & 22.8 & 849 & 24.8 & 1115 & \\
\hline $20-29$ & 37.7 & 1405 & 40.6 & 1829 & \\
\hline $30-39$ & 25.9 & 963 & 23.1 & 1042 & \\
\hline $40-49$ & 13.6 & 508 & 11.5 & 519 & \\
\hline Education & & & & & $<0.001$ \\
\hline Never & 29.8 & 1107 & 24.1 & 1102 & \\
\hline Primary & 35.3 & 1314 & 33.3 & 1524 & \\
\hline Secondary* & 19.8 & 737 & 24.3 & 1114 & \\
\hline Post-secondary** & 15.1 & 560 & 18.3 & 835 & \\
\hline Region & & & & & $<0.001$ \\
\hline Tigray & 15.0 & 559 & 14.4 & 662 & \\
\hline Afar & 3.3 & 124 & 1.9 & 88 & \\
\hline Amhara & 18.7 & 696 & 19.7 & 909 & \\
\hline Oromiya & 23.5 & 877 & 23.6 & 1089 & \\
\hline Somali & 2.2 & 81 & 2.2 & 100 & \\
\hline BG & 2.2 & 83 & 1.8 & 84 & \\
\hline SNNP & 19.8 & 739 & 23.5 & 1083 & \\
\hline Gamella & 0.8 & 30 & 0.8 & 35 & \\
\hline Harari & 0.8 & 28 & 0.6 & 27 & \\
\hline Addis Ababa & 13.0 & 485 & 11.1 & 511 & \\
\hline Dire Dawa & 0.6 & 23 & 0.5 & 23 & \\
\hline
\end{tabular}

* Secondary includes: Secondary (ET) and 'O' Level, 'A' Level (UG)

** Post-secondary includes: Technical, Higher (ET) and Tertiary, University (UG) 
Table C2. Differences in available sociodemographic characteristics between respondents and pooled confidantes in Uganda

\begin{tabular}{|c|c|c|c|c|c|}
\hline \multirow[b]{2}{*}{ Age } & \multicolumn{2}{|c|}{$\begin{array}{c}\text { Respondents } \\
(\mathrm{N}=2089)\end{array}$} & \multicolumn{2}{|c|}{$\begin{array}{l}\text { Pooled confidantes } \\
\qquad(\mathrm{N}=3268)\end{array}$} & \multirow{2}{*}{$\begin{array}{r}\mathrm{p} \text {-value } \\
<0.001\end{array}$} \\
\hline & & & & & \\
\hline $15-19$ & 22.4 & 467 & 17.6 & 542 & \\
\hline $20-29$ & 38.6 & 806 & 38.5 & 1184 & \\
\hline $30-39$ & 24.9 & 521 & 27.9 & 860 & \\
\hline $40-49$ & 14.1 & 295 & 16.0 & 491 & \\
\hline Education & & & & & $<0.001$ \\
\hline Never & 12.3 & 257 & 13.7 & 433 & \\
\hline Primary & 54.0 & 1127 & 44.5 & 1407 & \\
\hline Secondary* & 27.3 & 569 & 37.8 & 1195 & \\
\hline Post-secondary** & 6.5 & 135 & 4.0 & 125 & \\
\hline Region & & & & & $<0.001$ \\
\hline Central & 28.4 & 593 & 24.4 & 799 & \\
\hline Eastern & 24.2 & 506 & 23.4 & 765 & \\
\hline Northern & 25.3 & 529 & 26.2 & 855 & \\
\hline Western & 22.1 & 461 & 26.0 & 849 & \\
\hline
\end{tabular}

* Secondary includes: Secondary (ET) and 'O' Level, 'A' Level (UG)

** Post-secondary includes: Technical, Higher (ET) and Tertiary, University (UG) 


\section{Appendix D. Transmission bias adjustment calculations for abortion incidence and IUD/implant use estimates}

In order to adjust for the likelihood that respondents do not have perfect knowledge of their confidantes' abortions (Assumption 2), we calculated a transmission bias adjustment factor. Tables D1 and D2 show these calculations. Respondents with any confidantes who also self-reported an abortion were asked whether they told each of their confidantes about their abortion. We then calculated the proportion of respondents who told Confidantes 1, 2, and 3 (Table D1). This is done separately for each confidante to account for the likelihood that sharing is most common between the respondent and Confidante 1 . We then compute an adjustment factor for the confidante abortion estimates that is the inverse of this proportion. For example, in Ethiopia 53\% of respondents who self-reported an abortion told their Confidante 1 about this abortion. The adjustment factor is 1.0/0.53, which is 1.88 . Given that these relationships are meant to be reciprocal, we would interpret this to mean for every Confidante 1 abortion reported by a respondent, there is an additional 0.88 abortions that are not being reported. We then apply the confidante-specific adjustment factors to inflate the reported abortions in the past year separately among Confidantes 1, 2, and 3. To get the one-year transmission bias adjusted abortion incidence estimates among all confidantes, we sum the adjusted number of abortions for each confidante, then divide by the total number of confidantes, and multiply this estimate by 1,000 (Table D2). We conduct a similar procedure for calculating transmission bias adjusted estimates of current intrauterine device and implant use (Tables D3 and D4).

Table D1. Determining the transmission bias correction factor and adjusted number of confidante abortions from respondents who reported sharing their abortion experiences with each confidante in Ethiopia and Uganda

\begin{tabular}{|c|c|c|c|c|c|c|}
\hline & \multicolumn{4}{|c|}{$\begin{array}{l}\text { Among respondents who self-reported an } \\
\text { abortion and had any confidantes } \\
\text { (Ethiopia } n=97 ; \text { Uganda } n=97)\end{array}$} & \multicolumn{2}{|c|}{$\begin{array}{c}\text { Among respondents with any } \\
\text { confidantes } \\
\text { (Ethiopia } n=2,783 ; \text { Uganda } n=1,684)\end{array}$} \\
\hline & \multirow{2}{*}{$\begin{array}{l}\text { Number of } \\
\text { respondents } \\
\text { self-reporting } \\
\text { abortion with } \\
\text { a Confidante } \\
\text { X }\end{array}$} & \multicolumn{2}{|c|}{$\begin{array}{l}\text { Respondents who } \\
\text { told confidante X } \\
\text { about their } \\
\text { abortion }\end{array}$} & \multirow{2}{*}{$\begin{array}{l}\text { Correction } \\
\text { factor* } * *\end{array}$} & \multirow{2}{*}{$\begin{array}{c}\text { Respondent } \\
\text { reported \# of } \\
\text { confidante X } \\
\text { abortions in past } \\
12 \text { months }\end{array}$} & \multirow{2}{*}{$\begin{array}{c}\text { Transmission } \\
\text { bias adjusted \# } \\
\text { of confidante } \\
\text { abortions in past } \\
12 \text { months } \$ \\
\end{array}$} \\
\hline & & $\mathrm{N}$ & $\%$ & & & \\
\hline \multicolumn{7}{|l|}{ Ethiopia } \\
\hline Confidante \#1 & 97 & 55 & 0.53 & 1.88 & 26 & 49 \\
\hline Confidante \#2 & 41 & 17 & 0.42 & 2.40 & 14 & 34 \\
\hline Confidante \#3 & 20 & 8 & 0.57 & 1.76 & 2 & 4 \\
\hline \multicolumn{7}{|l|}{ Uganda } \\
\hline Confidante \#1 & 97 & 57 & 0.49 & 2.03 & 74 & 150 \\
\hline Confidante \#2 & 65 & 33 & 0.53 & 1.89 & 31 & 58 \\
\hline Confidante \#3 & 34 & 16 & 0.45 & 2.23 & 19 & 42 \\
\hline
\end{tabular}

*The correction factor is calculated as the inverse of the proportion of respondents who reported their abortions to the confidante

**Correction factor may not equal ( $1 / \%$ who told confidante about abortion their abortion) due to rounding $\S$ The transmission bias adjusted number of confidante abortions in the past 12 months is calculated by multiplying the respondent reported number of confidante abortions in the past 12 months by the correction factor 
Table D2. Determining the transmission bias adjusted one-year confidante abortion incidence rates per 1,000 women aged 15-49 in Ethiopia and Uganda

\begin{tabular}{|l|c|c|c|c|c|}
\hline & $\begin{array}{c}\text { Total transmission bias } \\
\text { adjusted confidante } \\
\text { abortions in past } \\
12 \text { months* }\end{array}$ & $\begin{array}{c}\text { Total number } \\
\text { of confidantes }\end{array}$ & $\begin{array}{c}\text { Transmission bias } \\
\text { adjusted one-year } \\
\text { abortion rate** } \S\end{array}$ & \multicolumn{2}{|c|}{$95 \%$ CIs } \\
\hline Ethiopia & 86 & 4612 & 18.6 & 12.0 & 28.8 \\
\hline Uganda & 250 & 3268 & 76.8 & 61.7 & 95.4 \\
\hline
\end{tabular}

*Total transmission bias adjusted confidante abortions in past 12 months reflect the sum of the transmission bias adjusted confidante abortions in past 12 months across all three confidantes in Table D1.

**Transmission bias adjusted one-year abortion rates calculated by dividing the total number of transmission bias adjusted confidante abortions in the past 12 months by the total number of confidantes

$\S$ Weighted using post-stratifications weights

Table D3. Determining the transmission bias correction factor and adjusted number of confidantes who currently use an IUD/implant from respondents who reported sharing their own IUD/implant use with each confidante in Ethiopia and Uganda

\begin{tabular}{|c|c|c|c|c|c|c|}
\hline & \multicolumn{4}{|c|}{$\begin{array}{c}\text { Among respondents who self-reported being a } \\
\text { current IUD/implant user and had any } \\
\text { confidantes } \\
\text { (Ethiopia } \mathrm{n}=231 ; \text { Uganda } \mathrm{n}=130)\end{array}$} & \multicolumn{2}{|c|}{$\begin{array}{l}\text { Among respondents with any } \\
\text { confidantes } \\
\text { (Ethiopia } n=2,783 ; \text { Uganda } n=1,684 \text { ) }\end{array}$} \\
\hline & \multirow{2}{*}{$\begin{array}{l}\text { Number of } \\
\text { respondents } \\
\text { self-reporting } \\
\text { IUD/implant } \\
\text { use with a } \\
\text { Confidante X } \\
\end{array}$} & \multicolumn{2}{|c|}{$\begin{array}{l}\text { Respondents who } \\
\text { told confidante } X \\
\text { about their } \\
\text { IUD/implant use }\end{array}$} & \multirow{2}{*}{$\begin{array}{l}\text { Correction } \\
\text { factor* }^{* *}\end{array}$} & \multirow{2}{*}{$\begin{array}{l}\text { Respondent } \\
\text { reported \# of } \\
\text { confidante X who } \\
\text { currently use an } \\
\text { IUD/implant }\end{array}$} & \multirow{2}{*}{$\begin{array}{c}\text { Transmission } \\
\text { bias adjusted \# } \\
\text { of confidantes } \\
\text { who currently } \\
\text { use an } \\
\text { IUD/implant§ }\end{array}$} \\
\hline & & $\mathrm{N}$ & $\%$ & & & \\
\hline Confidante \#1 & 231 & 201 & 0.88 & 1.14 & 511 & 582 \\
\hline Confidante \#2 & 113 & 78 & 0.65 & 1.53 & 196 & 299 \\
\hline Confidante \#3 & 47 & 28 & 0.54 & 1.87 & 74 & 138 \\
\hline Confidante \#1 & 130 & 111 & 0.86 & 1.17 & 363 & 424 \\
\hline Confidante \#2 & 87 & 60 & 0.71 & 1.42 & 211 & 299 \\
\hline Confidante \#3 & 42 & 29 & 0.76 & 1.32 & 142 & 187 \\
\hline
\end{tabular}

*The correction factor is calculated as the inverse of the proportion of respondents who reported their abortions to the confidante

**Correction factor may not equal (1/\% who told confidante about abortion their abortion) due to rounding $\S$ The transmission bias adjusted number of confidante abortions in the past 12 months is calculated by multiplying the respondent reported number of confidante abortions in the past 12 months by the correction factor 
Table D4. Determining the transmission bias adjusted prevalence estimate for currently IUD/implant use in Ethiopia and Uganda

\begin{tabular}{|l|c|c|c|c|c|}
\hline & $\begin{array}{c}\text { Total transmission bias } \\
\text { adjusted number of } \\
\text { confidantes who are current } \\
\text { IUD/implant users* }\end{array}$ & $\begin{array}{c}\text { Total number } \\
\text { of confidantes }\end{array}$ & $\begin{array}{c}\text { Transmission bias } \\
\text { adjusted current } \\
\text { IUD/implant use } \\
\text { prevalence } \\
\text { estimate**§ }\end{array}$ & \multicolumn{2}{|c|}{$95 \%$ CIs } \\
\hline Ethiopia & 1020 & 4612 & 22.1 & 19.1 & 25.5 \\
\hline Uganda & 910 & 3268 & 27.8 & 23.3 & 33.0 \\
\hline
\end{tabular}

*Total transmission bias adjusted number of confidantes who are current IUD/implant users reflect the sum of the transmission bias adjusted number of confidantes who currently use an IUD/implant in Table D3

**Transmission bias adjusted current IUD/implant use prevalence estimate calculated by dividing total transmission bias adjusted number of confidantes who are current IUD/implant users by the total number of confidantes $\S$ Weighted using post-stratifications weights 